\title{
Galaxy gas as obscurer: II. Separating the galaxy-scale and nuclear obscurers of Active Galactic Nuclei
}

\author{
Johannes Buchner ${ }^{1,2 \star}$, Franz E. Bauer ${ }^{1,2,3}$ \\ ${ }^{1}$ Millenium Institute of Astrophysics, Vicuña. MacKenna 4860, 7820436 Macul, Santiago, Chile \\ ${ }^{2}$ Pontificia Universidad Católica de Chile, Instituto de Astrofísica, Casilla 306, Santiago 22, Chile \\ ${ }^{3}$ Space Science Institute, 4750 Walnut Street, Suite 205, Boulder, Colorado 80301
}

Accepted XXX. Received YYY; in original form ZZZ

\begin{abstract}
The "torus" obscurer of Active Galactic Nuclei (AGN) is poorly understood in terms of its density, substructure and physical mechanisms. Large X-ray surveys provide model boundary constraints, for both Compton-thin and Compton-thick levels of obscuration, as obscured fractions are mean covering factors $f_{\text {cov }}$. However, a major remaining uncertainty is host galaxy obscuration. In Paper I we discovered a relation of $N_{\mathrm{H}} \propto M_{\star}^{1 / 3}$ for the obscuration of galaxy-scale gas. Here we apply this observational relation to the AGN population, and find that galaxy-scale gas is responsible for a luminosity-independent fraction of Compton-thin AGN, but does not produce Compton-thick columns. With the host galaxy obscuration understood, we present a model of the remaining, nuclear obscurer which is consistent with a range of observations. Our radiation-lifted torus model consists of a Compton-thick component $\left(f_{\text {cov }} \sim 35 \%\right)$ and a Compton-thin component $\left(f_{\text {cov }} \sim 40 \%\right)$, which depends on both black hole mass and luminosity. This provides a useful summary of observational constraints for torus modellers who attempt to reproduce this behaviour. It can also be employed as a sub-grid recipe in cosmological simulations which do not resolve the torus. We also investigate host-galaxy X-ray obscuration inside cosmological, hydrodynamic simulations (EAGLE, Illustris). The obscuration from ray-traced galaxy gas can agree with observations, but is highly sensitive to the chosen feedback assumptions.
\end{abstract}

\section{INTRODUCTION}

The vast majority of Active Galactic Nuclei (AGN) are obscured by thick columns of gas and dust. X-ray surveys over the last decade indicate that $20-40 \%$ are hidden behind Compton-thick column densities $\left(N_{\mathrm{H}} \gtrsim 10^{24} \mathrm{~cm}^{-2}\right)$ and of the remaining population, $\sim 75 \%$ are obscured with $N_{\mathrm{H}}=10^{22}-10^{24} \mathrm{~cm}^{-2}$ (e.g. Treister et al. 2004; Brightman et al. 2014; Ueda et al. 2014; Buchner et al. 2015; Aird et al. 2015 ) at the peak of AGN activity at redshift $z=0.5-3$. An open question is whether the same gas reservoir is responsible for fuelling the AGN by accretion onto Supermassive Black Holes (SMBHs), and whether it itself is affected by AGN activity. To address this, the first step is to identify the scale at which the obscuring gas resides. Traditionally, AGN obscuration is associated with the "torus", a nuclear ( 10pc) structure surrounding the accretion disk. Many basic questions about this gas reservoir remain to be answered, including its density, substructure and stability mechanism (Elitzur 2006; Hoenig 2013). Assuming sampling from random viewing angles, the high fraction of obscured AGN implies high covering fractions. Turbulent structures such as winds from accretion disks have been invoked to explain this (Krolik \& Begelman 1988). However, for the covering fractions to be useful constraints for torus models, the im- portance of galaxy-scale gas to the obscuration has to be estimated. Separating the covering and column densities from nuclear and galaxy-scale obscurers is the goal of this work.

Local galaxies exemplify that several scales can contribute to the obscured columns. The Milky Way gas distribution shows column densities of $N_{\mathrm{H}}>10^{22} \mathrm{~cm}^{-2}$, but only at very low Galactic latitudes $\left(|b| \lesssim 2^{\circ}\right.$, Dickey \& Lockman 1990; Kalberla et al. 2005). Towards the Galactic Centre, columns with $N_{\mathrm{H}}>10^{24} \mathrm{~cm}^{-2}$ can be found in the Central Molecular Zone (Morris \& Serabyn 1996; Molinari et al. 2011) and in the equivalent central zones of nearby AGN host galaxies (Prieto et al. 2014). Also the obscuration in the AGN host galaxy NGC 1068 is clearly nuclear (in this work: $\sim 100$ pc or smaller), because its Compton-thick column is observed in a face-on galaxy (Matt 1997). On the other hand, many nearby, obscured AGN are hosted in edgeon galaxies (Maiolino \& Rieke 1995), which suggests that dust-lanes may also be important obscurers (see also Goulding \& Alexander 2009, for galactic optical/infrared extinction). Hence Matt (2000) proposed a two-phase model for the obscuration of AGN: a central, nuclear obscurer which provides Compton-thick obscuration, and the host galaxy, which provides mildly obscured lines of sight.

However, it has remained difficult to quantifying the 
covering fractions of host galaxy-scale gas. The Milky Way and local galaxies are limited in their use as templates for the high-redshift universe, specifically at peak SMBH growth $(z=0.5-3$; e.g., Ueda et al. 2003; Aird et al. 2010). At that time, the gas content in galaxies was probably higher, as indicated by molecular gas measurements (e.g. Tacconi et al. 2013). To observationally decouple the galaxy-scale and nuclear X-ray obscurer, we need to go beyond a single, central source.

This work builds on observational results from Paper I of the obscuring column distribution of galaxy-scale gas alone. This stellar-mass dependent result was derived from the X-ray spectra of an unbiased Gamma Ray Burst (GRB) sample. In Section 2 we present our computation of transferring these results from the GRB host population to the AGN host population, taking into account the different stellar mass distribution. Section 3 presents our prediction of the galaxy-scale obscurer alone and compared with obscured fractions from AGN surveys. Independently, Section 4 looks into simulated galaxies in hydro-dynamic cosmological simulations. These also provide predictions for the amount of gas inside galaxies, from which we derive obscured fractions using ray-tracing. We discuss the implications and limitations of our two approaches in Section 5.1. We then subtract the galaxy-scale obscurer from the observed obscuration and reveal the remaining, nuclear obscurer in Section 5.2. Its luminosity-dependent behaviour is analysed in detail, for which we present a model in Section 6 .

\section{METHODOLOGY}

Our goal is to predict the fraction of obscured AGN from the obscuration of host galaxy-scale gas alone, i.e. without nuclear obscuration (the torus and central molecular zones). In this fashion we will be able to separate the large-scale and small-scale obscurer. In Paper I we established a relation between the distribution of X-ray absorbing column densities, $N_{\mathrm{H}}$, in galaxies and the stellar mass of the galaxy. It follows approximately a log-normal distribution around

$N_{\mathrm{H}}=10^{21.7} \mathrm{~cm}^{-2} \times\left(M_{\star} / 10^{9.5} M_{\odot}\right)^{1 / 3}$

with standard deviation $\sigma=0.5$ dex. This $N_{\mathrm{H}}-M_{\star}$ relation was derived using an unbiased sample of long Gamma Ray Bursts (LGRBs). Because the obscuration is host mass dependent, the obscurer is arguably the host galaxy itself. In Paper I we show that modern cosmological hydro-dynamic simulations reproduce absorption by galaxy-scale metal gas and predict $N_{\mathrm{H}}-M_{\star}$ relations very similar to Equation 1 .

We now apply this relation to the AGN host galaxy population to estimate host galaxy obscuration. This relation was derived from actively star forming galaxies, therefore a caveat is that results may slightly over-represent the galaxy gas present in AGN host galaxies which have average (Rosario et al. 2011, 2013; Santini et al. 2012) or even below-average (Mullaney et al. 2015) star formation rates. The major benefit of using this relationship is that it is based on the same observable as AGN obscured fractions, namely the photo-electric absorption of X-rays. Paper I also investigated the host galaxy metallicity bias of LGRB and concluded that it has a negligible effect on the obscurer. Local galaxies are also shown there to follow the $N_{\mathrm{H}}-M_{\star}$ relation.
In Section 4 we further test whether our assumptions hold using simulations.

We begin with the stellar mass function (SMF) of the galaxy population. Its shape $P\left(M_{\star} \mid z\right)$ is approximately a Schechter function and was measured by e.g. Muzzin et al. (2013) and Ilbert et al. (2013) out to $z \sim 4$. Then we populate the galaxies with AGN. The occupation probability $P\left(\mathrm{AGN} \mid M_{\star}, z\right)$ has been measured by Aird et al. (2012) and Bongiorno et al. (2012) primarily for the redshift interval $z=0.5-2$. More accurately, these authors measure the specific accretion rate distribution (SARD), $P\left(L_{\mathrm{X}} \mid M_{\star}, z\right)$, where $L_{\mathrm{X}}$ is the $2-10 \mathrm{keV} \mathrm{X}$-ray luminosity. They find factorised powerlaw relationships of the form $P\left(L_{\mathrm{X}} \mid M_{\star}, z\right)=A \cdot L_{\mathrm{X}}^{\gamma_{L}} \cdot M_{\star}^{\gamma_{M}} \cdot(1+z)^{\gamma_{z}}$. At the highest luminosities, an Eddington limit is required to explain the steep decrease at the bright end of the luminosity function (Aird et al. 2013). In this work, however, we focus on the $L_{\mathrm{X}}=10^{42-45} \mathrm{erg} / \mathrm{s}$ luminosity range and thus use only the observed relation. The final ingredient is the obscuring column density distribution $P\left(N_{\mathrm{H}} \mid M_{\star}\right)$, which is given by Equation 1 as a log-normal distribution. We assume that the galaxy-scale gas is independent of nuclear activity for individual galaxies.

The obscured fraction can then be simply computed by Monte Carlo simulations. Analytically we can put the three distributions together as

$$
P\left(N_{\mathrm{H}}, L_{\mathrm{X}}, M_{\star} \mid z\right)=\underbrace{P\left(M_{\star} \mid z\right)}_{\mathrm{SMF}} \times \underbrace{P\left(L_{\mathrm{X}} \mid M_{\star}, z\right)}_{\mathrm{SARD}} \times \underbrace{P\left(N_{\mathrm{H}} \mid M_{\star}\right)}_{\mathrm{Eq} 1}
$$

After inserting the factorised powerlaw relationship of the $\mathrm{SARD}$, the result has the form

$$
P\left(N_{\mathrm{H}}, L_{\mathrm{X}}, M_{\star} \mid z\right)=A \times L_{X}^{\gamma_{L}} \times f\left(M_{\star} \mid z\right) \times P\left(N_{\mathrm{H}} \mid M_{\star}\right) .
$$

The SARD implies that while the absolute probability of finding an AGN is a function of luminosity, the mass distribution is independent of luminosity. That is, at every luminosity (not considering the Eddington limit), the same mix of host stellar masses contribute. Therefore, the implication is that the galaxy-scale gas obscuration is the same at all AGN luminosities. An Eddington-limit-like effect, i.e. the suppression of low-mass galaxies at the bright end, in combination with the $N_{\mathrm{H}}-M_{\star}$ relation should induce a positive correlation between luminosity and obscured fraction. Instead, a negative correlation is observed: bright AGN are less frequently obscured (Ueda et al. 2003; Hasinger et al. 2005; La Franca et al. 2005; Ebrero et al. 2009; Ueda et al. 2014; Buchner et al. 2015; Aird et al. 2015). We therefore retain the simple, luminosity-independent formalism.

We adopt an AGN definition of $L_{\mathrm{X}}>10^{42} \mathrm{erg} / \mathrm{s}$. The frequency distribution of column densities $N_{\mathrm{H}}$ for the AGN population is then computed by integrating over stellar mass and luminosity:

$P\left(N_{\mathrm{H}} \mid z\right)=\iint_{10^{42} \mathrm{erg} / \mathrm{s}}^{\infty} P\left(N_{\mathrm{H}}, L_{\mathrm{X}}, M_{\star} \mid z\right) d L_{\mathrm{X}} d M_{\star}$.

Finally the obscured AGN fraction is the cumulative distribution, i.e. the fraction of AGN hidden behind a certain 


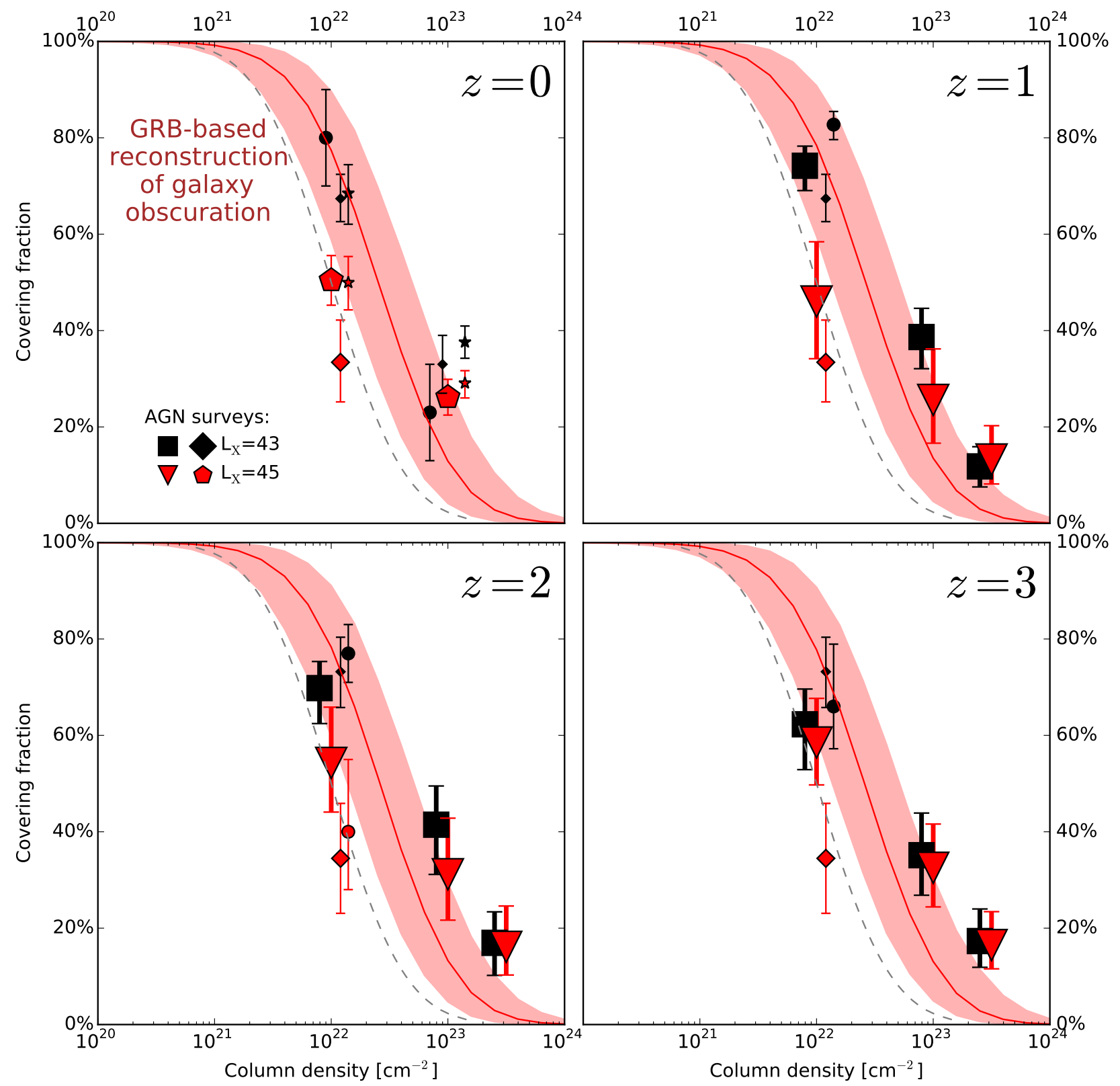

Figure 1. Galaxy-scale obscuration of AGN. At various redshift intervals we show the fraction of AGN (y-axis) that is covered by a given column density $N_{\mathrm{H}}$ (x-axis). The red curve indicates our derivations based on GRB tomography with uncertainties shown in red shading. Galaxy-scale obscuration is negligible for column densities of $10^{24} \mathrm{~cm}^{-2}$, but is an important contributor to the AGN fraction at $N_{\mathrm{H}} \approx 10^{22-23} \mathrm{~cm}^{-2}$. Data points represent intrinsic (flux bias-corrected) obscured fractions in the Compton-thin population from AGN surveys: Bright AGN $\left(L(2-10 \mathrm{keV}) \approx 10^{45} \mathrm{erg} / \mathrm{s}\right.$, red error bars) show lower obscurations than faint AGN $\left(L(2-10 \mathrm{keV}) \approx 10^{43} \mathrm{erg} / \mathrm{s}\right.$, black error bars). The data points are diamonds, circles, star small symbols (Ueda et al. 2014; Aird et al. 2015; Ricci et al. 2016, respectively) and pentagons and squares/triangles larger symbols (Burlon et al. 2011; Buchner et al. 2015, respectively). Because AGN also have a nuclear obscurer, these should be regarded as upper limits for galaxy gas obscuration (red shading). Arguably below all data constraints is a normal distribution around $10^{22} \mathrm{~cm}^{-2}$ (dashed grey curve), which is kept constant across panels.

column density threshold $N_{\mathrm{H}}$ or higher:

$f_{\text {cov }}\left(>N_{\mathrm{H}}\right)=\int_{N_{\mathrm{H}}}^{\infty} P\left(N_{\mathrm{H}}{ }^{\prime} \mid z\right) d N_{\mathrm{H}}{ }^{\prime}$.

Into the calculation of $f_{\text {cov }}$ we propagate the uncertainties from the obscuration relation for Paper I. We consider two SMF (Muzzin et al. 2013 and Ilbert et al. 2013) and two SARD measurements (Aird et al. 2012 and Bongiorno et al. 2012), to incorporate systematic uncertainties. To summar- ise, we rely only on observational relations to predict the obscuration of the AGN population by galaxy-scale gas.

\section{RESULTS}

The obscured fraction of the AGN population from combining the observed relationships is shown in Figure 1. Each panel represents a specific redshift. The red curve 
shows our fraction of obscured AGN (y-axis) for a given column density $N_{\mathrm{H}}$ (x-axis). Red shading shows the uncertainties $(2 \sigma)$, which are dominated by the $N_{\mathrm{H}}-M_{\star}$ relation $( \pm 0.2 \mathrm{dex})$, while our marginalisation over different SARD and SMF moves the $N_{\mathrm{H}}$ distribution by less than 0.08 dex and 0.04 dex, respectively (not depicted).

Firstly, galaxy-scale gas does not provide Comptonthick column densities $\left(N_{\mathrm{H}}>\sigma_{T}^{-1}=1.5 \times 10^{24} \mathrm{~cm}^{-2}\right)$. This is because massive galaxies which reach those densities are rare and therefore represent a negligible fraction of the AGN population. In contrast, the observed fraction of Comptonthick AGN is 38\% (e.g. Buchner et al. 2015; Ricci et al. 2016; Aird et al. 2015). We can conclude that Compton-thick obscuration is always associated with the nuclear region. An alternative, theoretical argument based on the total metal gas mass present in galaxies is laid out in Appendix B and arrives at the same conclusion.

We therefore focus on the Compton-thin obscurer. This step implicitly assumes that the Compton-thick nuclear obscurer is randomly oriented with respect to the galaxy, in accordance with chaotic accretion (King \& Pringle 2006). We now compare with measurements of the obscured fraction of Compton-thin AGN from surveys. When these fractions are treated as covering fractions, they contain both galaxyscale obscuration and nuclear obscuration. Therefore the data points should always be understood as upper limits to the galaxy-scale gas obscuration. Figure 1 shows results from surveys at the peak of the accretion history $(z=1-3$ panels, Ueda et al. 2014; Buchner et al. 2015; Aird et al. 2015) and surveys which include the local Universe (Burlon et al. 2011; Ueda et al. 2014; Aird et al. 2015; Ricci et al. 2016). Results are often given as the intrinsic fraction of Comptonthin AGN with $N_{\mathrm{H}}>10^{22} \mathrm{~cm}^{-2}$, a common definition of "obscured" AGN. When comparing our results with these data points in Figure 1, we find that galaxy-scale obscuration is likely the dominant contributor at $N_{\mathrm{H}}=10^{22-23} \mathrm{~cm}^{-2}$. Two cases are important: (1) the fraction of obscured AGN at the bright end $\left(L_{\mathrm{X}} \geq 10^{45} \mathrm{erg} / \mathrm{s}\right)$, where the lowest obscured fractions are observed $\left(f_{\text {bright }} \sim 40 \%\right.$, shown as red data points), and (2) at the faint end $\left(L_{\mathrm{X}} \sim 10^{43} \mathrm{erg} / \mathrm{s}\right)$, where the highest obscured fractions are observed $\left(f_{\text {faint }} \sim 70 \%\right.$, shown in black). Our results for galaxy-scale obscuration are, as discussed in Section 2, luminosity-independent. Because of the large uncertainties, we cannot distinguish whether galaxy-scale obscuration decreases toward the bright end, or increases toward the faint end. In Section 5 we also examine systematic uncertainties from the enhanced star formation rates of LGRB hosts compared to AGN hosts. In any case, however, our prediction indicates that galaxy-scale obscuration is sufficient to explain the observed fractions of obscured AGN at $N_{\mathrm{H}}=10^{22-23} \mathrm{~cm}^{-2}$.

At higher column densities the observed obscured fractions lie systematically higher than our results from galaxyscale gas. Therefore a nuclear obscurer is required to explain this obscuration excess and becomes dominant at approximately $N_{\mathrm{H}} \approx 10^{23.5} \mathrm{~cm}^{-2}$. The shape of the distribution is driven by the Gaussian distribution of the $N_{\mathrm{H}}-M_{\star}$ relation, the distribution adopted in Paper I to fit the dispersion of LGRB LOS column densities. Adopting a different distribution would result in the same width, but may allow flatter tails and permit more unobscured LOS.

We emphasise that our results are meaningful for the
AGN population - the obscuration of individual host galaxies is stellar-mass dependent with substantial variations between individual galaxies (see Equation 1).

\section{COSMOLOGICAL HYDRO-DYNAMIC SIMULATIONS}

In this section we assess the gas content in simulated galaxies. This allows us to validate our approach and to compare with the predictions for galaxy-scale obscuration by those simulations. Modern cosmological hydro-dynamic simulations self-consistently evolve galaxies and their processes (star formation, gas accretion, supernova and AGN feedback, etc.) in the context of well-constrained $\Lambda \mathrm{CDM}$ cosmologies. These simulations use the initial conditions of the baryon density available in the early Universe and are tuned to reproduce the local galaxy population. We consider two state-of-the-art cosmological hydro-dynamic simulations which also produce realistic galaxy morphologies. These simulations allow us to look at the spatial distribution of gas inside galaxies.

\subsection{Simulation sets: EAGLE \& Illustris}

The Evolution and Assembly of Galaxies and their Environment (EAGLE) simulation (Schaye et al. 2015; Crain et al. 2015) reproduces many observed quantities; it reproduces very well the stellar mass function (Furlong et al. 2015b) and size distribution (Furlong et al. 2015a) of galaxies as a function of cosmic time, being calibrated to reproduce these at $z=0$. Further relevant for this work, it also produces galaxies with realistic galaxy morphologies (Schaye et al. 2015) and gas contents consistent with observations of $\mathrm{CO}$ and $\mathrm{HI}$ (Bahe et al. 2015) as well as $\mathrm{H}_{2}$ (Lagos et al. 2015). This encourages us to look inside simulated galaxies and assess the obscuration provided by them. EAGLE includes black hole particles, which are seeded into dark matter halos exceeding masses of $10^{10} M_{\odot}$. These black holes are kept near the galaxy centre and may accrete when gas is nearby, in turn activating a heating feedback mechanism (Schaye et al. 2015). The strength of EAGLE lies in its minimalistic subgrid recipes and the systematic exploration of alterations. Besides the reference model (L0100N1504_REFERENCE) which we use primarily, a series of simulations with parameter variations have been run and analysed. These explore the impact of the type and strength of supernova feedback, AGN heating and criteria for when stars are formed (Crain et al. 2015).

We also consider Illustris (Vogelsberger et al. 2014b,a), another hydro-dynamic cosmological simulation. This simulation also reproduces many observed quantities; most relevant for this work is that it reproduces the morphology of galaxies, the gas content from CO observations (Vogelsberger et al. 2014b; Genel et al. 2014). The Illustris sub-grid models were chosen in consideration of the stellar mass function, star formation history and mass-metallicity relation. However, the weak tuning provides only mediocre agreement with regards to the galaxy stellar mass function (Schaye et al. 2015) and size distribution (urlongFURLONG et al. 2015a). On the positive side, the Illustris simulation is based on the AREPO hydrodynamics code which has been shown to reproduce galaxy features well (e.g. Vogelsberger et al. 
2012; Nelson et al. 2013). The gas particle resolution in $\mathrm{Il}$ lustris is adaptive, with some cells being as small as $48 \mathrm{pc}$ in the highest resolution simulation (Illustris-1) used here, indicating that modern cosmological simulations indeed resolve galaxies into small sub-structures. Similar to EAGLE, Illustris includes black holes which are created and kept in the gravitational potential minimum of galaxies inside halos of mass $M_{\mathrm{DM}}>7.1 \times 10^{10} M_{\odot}($ Sijacki et al. 2015$)$.

\subsection{Ray tracing of gas}

We first investigate the gas distribution in the reference simulations. We focus on the metal component of gas as $\mathrm{O}$ and $\mathrm{Fe}$ are, for the relevant obscuring columns and redshifts discussed here, the most important elements for photoelectric absorption of X-rays. In galaxy evolution models, the massive end of the existing stellar population expels metals into the galaxy. The metal gas produced per stellar mass is determined by the chosen IMF and the metal yield, with the latter tuned to reproduce the stellar mass function (e.g. $\mathrm{Lu}$ et al. 2015). The total metal gas mass residing in galaxies further depends on the chosen feedback models which can expel gas out of the galaxy. The strength of feedback is also constrained by matching the stellar mass function and galaxy properties (e.g. the color distribution, Croton et al. 2006). Typically the metal gas mass inside galaxies follows a $M_{Z}: M_{\star}$ ratio between $1: 30$ and $1: 100$ in semi-analytic models at $z=0-3$ (e.g. Croton et al. 2006, 2016); Plots of these models can be found in Appendix B. The crucial remaining uncertainty is the arrangement of that gas inside galaxies, as the concentration of gas defines its column density.

We apply ray tracing starting from the most massive black hole particle of each simulated galaxy (subhalo). From that position, we radiate in random sightlines any metal gas bound to the subhalo. Each location on the ray is assigned the density from the nearest gas particle (Voronoi tesselation $)^{1}$. Finally the density is integrated to a total metal column density. We then compute an equivalent hydrogen column density distribution by adopting Wilms et al. (2000) local inter stellar medium (ISM) abundances. This mimics how $N_{\mathrm{H}}$ is derived in X-ray observations. We adopt $h=0.7$ and work in physical units at redshifts $z=0,1,2$ and 3 .

Using the covering fractions for each SMBH we compute the obscured fraction of the AGN population. Because reproducing the luminosity function from simulated black hole accretion is on-going research (see Sijacki et al. 2015; RosasGuevara et al. 2016, for Illustris and EAGLE respectively), we do not rely on the instantaneous accretion rates provided by the simulations. The effect of adopting these is discussed in Appendix A. Instead, for each galaxy we randomly assign a luminosity according to the SARD of Aird et al. $(2012)^{2}$ and use only those with $L(2-10 \mathrm{keV})>10^{42} \mathrm{erg} / \mathrm{s}$. The last step is repeated 400 times to increase the sample size. With

1 Our Voronoi tessellation ray tracing code can be found at https://github.com/JohannesBuchner/LightRayRider; Catalogues of the obscuration of all considered simulated galaxies are available from the first author on request.

${ }^{2}$ We use host galaxy stellar masses within twice the half-light radius. the column density distribution for each AGN available, we compute the obscured fraction as a function of column density $N_{\mathrm{H}}$ of the simulated population.

\subsection{Covering fraction of simulated galaxies}

We present the fraction of AGN showing column densities larger than a given $N_{\mathrm{H}}$ value in Figure 2. The plot is made in the same fashion as the previous observational Figure 1 . We find that both the EAGLE (thick curve) and Illustris (thin curve) simulations produce a negligible number of Comptonthick AGN through host-galaxy obscuration. This is consistent with the assumption that Compton-thick obscuration is associated with a nuclear obscuration in the unresolved vicinity of the black hole.

For comparison with observations, obscured fractions from AGN surveys are used, as before in Section 3. Downwards-pointing triangles in Figure 2 indicate constraints for the obscured fraction of luminous, Comptonthin AGN. Arbitrary additional nuclear obscuration may be included in them, therefore they should be interpreted as upper limits to the galaxy-scale obscuration. We find that the Illustris simulation (thin curve) fulfils these constraints, as it always produces covering fractions below $40 \%$ for columns of $N_{\mathrm{H}} \geq 10^{22} \mathrm{~cm}^{-2}$. In contrast, the EAGLE reference simulation (thick curve) produces an excess of obscured AGN at $N_{\mathrm{H}}=10^{22} \mathrm{~cm}^{-2}$ at redshifts $z=1-3$ in Figure 2. This is in violation of observations even when the higher data point from low-luminosity AGN is considered. At higher column densities, the large-scale galaxy gas of the EAGLE simulation produces covering fractions consistent with the observations, with no need for a nuclear obscurer up to $N_{\mathrm{H}}=10^{23.5} \mathrm{~cm}^{-2}$. In contrast, Illustris galaxies do not provide column densities of $N_{\mathrm{H}}>10^{23} \mathrm{~cm}^{-2}$ and thus require a nuclear obscurer to explain those observations. At redshift $z=0$, both EAGLE and Illustris are consistent with the data points.

To summarise, the covering fractions show a similar behaviour as our observational results presented in Section 3. Galaxy-scale obscuration is an important obscurer at column densities $N_{\mathrm{H}}=10^{22} \mathrm{~cm}^{-2}$. The differences between EAGLE and Illustris simulations can be explained by the sub-grid physics adopted. Appendix A investigates sub-grid physics variations in detail. In general, weaker feedback implementations lead to very high covering factors. Therefore, Appendix A concludes that obscured fractions provide upper limits which may be used to rule out specific feedback implementations.

\subsection{Metallicity of sightlines to AGN and GRBs}

The metallicity inside galaxies may be different in GRB starformation sites and the nucleus of galaxies. Therefore, we now investigate the hydrogen column density of gas in the EAGLE $^{3}$ simulation and compute the metallicity of sightlines. We note that Bahe et al. (2015) found good agreement between the EAGLE galaxies and observations in terms of hydrogen masses and surface densities. We discuss in Paper

3 EAGLE subdivides gas in hydrogen, helium and individual metals, while Illustris only separates out neutral hydrogen. 


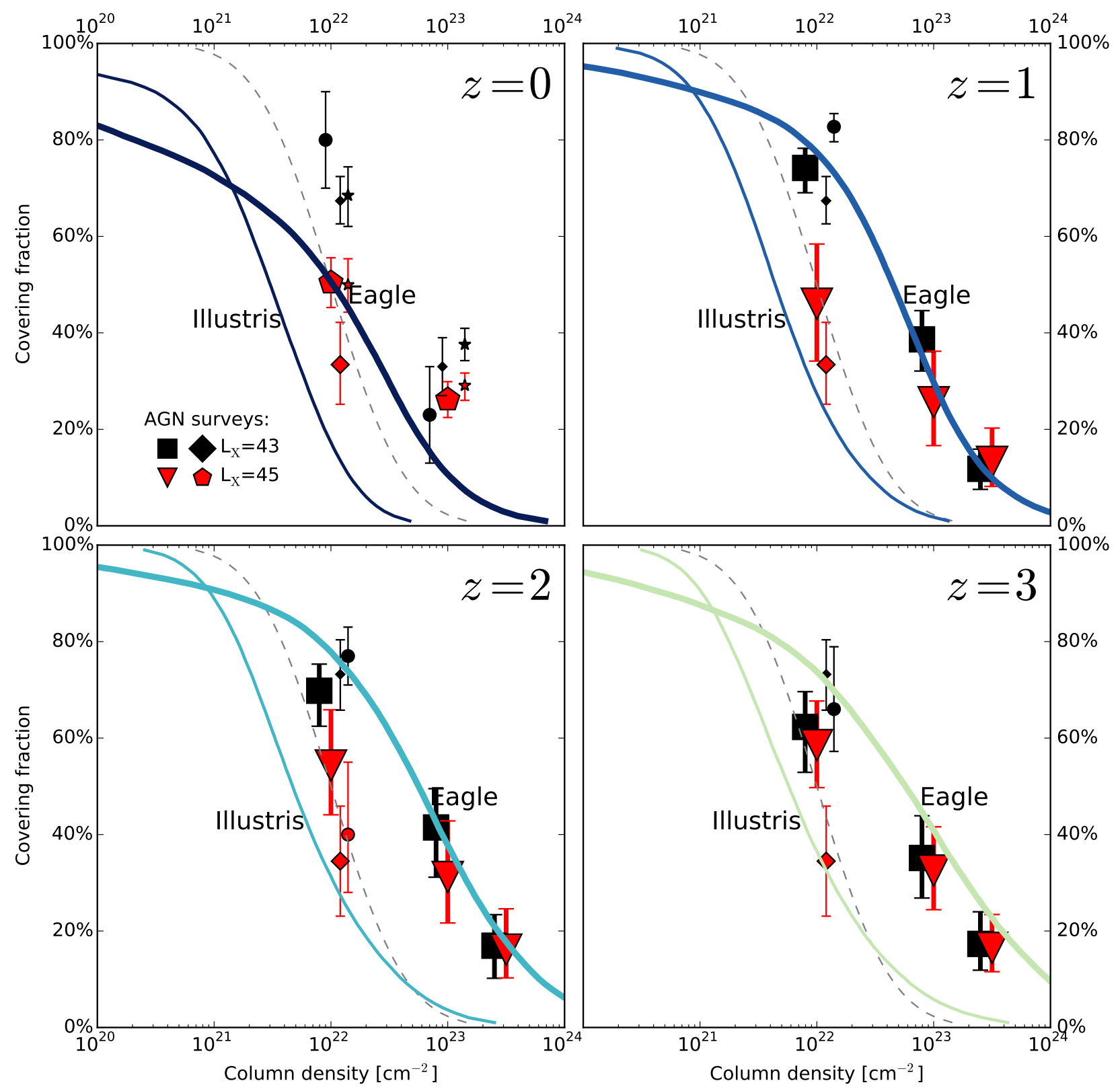

Figure 2. Hydro-dynamic cosmological simulation results for the galaxy-scale gas obscuration of AGN. At various redshift intervals (panels) we show the fraction of AGN (y-axis) that is covered by a given column density $N_{\mathrm{H}}$ (x-axis). Curves indicate results from ray-tracing the metal gas. The EAGLE reference simulation (thick line) produces thicker column densities than Illustris (thin line). Data points are the same as in Figure 1. These show fractions from surveys of bright/faint AGN in triangle/square symbols respectively. Because AGN also have a nuclear obscurer, these should be interpreted as upper limits for galaxy gas obscuration. The dashed grey curve (same as in Figure 1) is kept constant across panels for reference.

I that typical GRB sightlines are observationally consistent with the metal abundance of the local ISM.

Figure 3 shows that the LOS metal abundances are very similar for AGN and LGRBs. The metal abundance $N_{\mathrm{Z}} / N_{\mathrm{H}}$, normalised to Wilms et al. 2000 abundances is presented. For AGN (red squares), sightlines end at the central black hole. The conditions and sites of creation for LGRBs are not completely understood, however they trace the blue light in galaxies (Bloom et al. 2002; Fruchter et al. 2006). Under the simple assumption that LGRBs are created in star-forming regions of high hydrogen content, we start our ray-tracing from the region which is densest in hydrogen-gas in each galaxy. The corresponding sight-line abundances are shown as grey circles in Figure 3. If we further consider that LGRBs only occur in galaxies with stellar metallicities below solar, we arrive at the results presented by black circles. Such a metallicity cut is necessary to reproduce the mass and luminosity distribution of the observed GRB population, as well as observed metal emission lines (Hjorth \& Bloom 2012; Vergani et al. 2015; Krühler 2015; Perley et al. 2016c). Remarkably, the metallicity cut has little effect on the absorbing LOS metallicity. At all redshifts and stellar masses, we find 


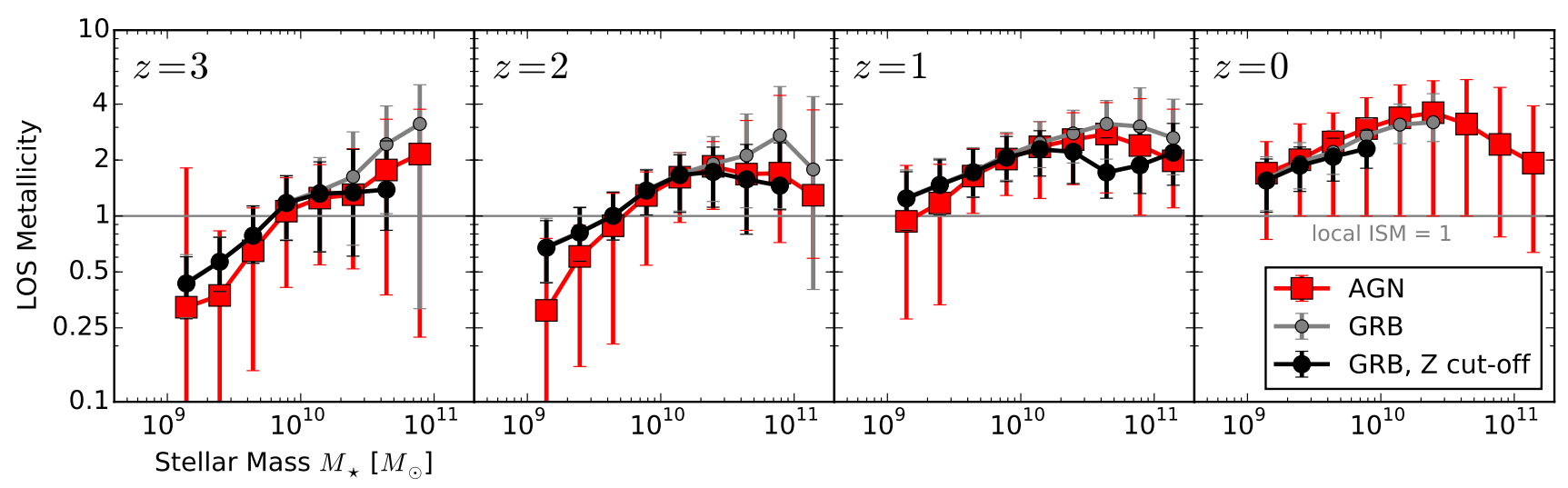

Figure 3. LOS column metal abundances. In the EAGLE reference simulation, hydrogen and metal gas densities were integrated along random sightlines from the central black hole to the edge of the host galaxy (AGN, red squares). The ratio is presented relative to local ISM abundances. For GRBs (grey circles), ray tracing starts from the hydrogen-densest regions. Black circles are the same, but only allow galaxies with sub-solar stellar metallicity as GRB hosts. Such a sub-selection is necessary to reproduce the mass and luminosity distribution of the observed GRB population (Hjorth \& Bloom 2012; Vergani et al. 2015; Krühler 2015; Perley et al. 2016c). Curves present the median, while the errorbars represent the $1 \sigma$ scatter in the population. LOS metallicities show a stellar-mass dependence and evolution over cosmic time, but are very similar for GRBs and AGN.

similar metal abundances between AGN and LGRB sightlines. In general, sightlines average across large parts of the galaxy, and thus are not affected by local metal variations. This confirms the assumptions of Section 3, where we applied host galaxy obscuration measured in LGRBs sightlines to the AGN population.

The abundance of metals along sightlines increases with stellar mass in Figure 3. In massive galaxies, a larger number of massive stars are available to pollute the ISM and the deeper galaxy potential can retain more metal gas. Metal abundances also increase over cosmic time, as metals build up inside galaxies. For AGN, the SARD skews the stellar mass distribution to the massive end. There, LOS abundances of about twice the local ISM metallicity are predicted at all considered redshifts. In contrast, LGRB host galaxies are predominantly found to have $M_{\star} \approx 10^{9} M_{\odot}$ at $z \sim 2$ (see e.g., Schulze et al. 2015; Perley et al. 2016b,c). Therefore sub-solar metallicities are to be expected in LGRB afterglow spectroscopy. For research on the optical absorption of LGRBs by HI, we refer interested readers to the simulations of Pontzen et al. (2010).

\section{DISCUSSION}

\subsection{AGN are obscured by their host galaxy}

Our main result is that the host galaxy gas provides a luminosity-independent obscurer, for which we compute covering fractions. This obscurer does not have Compton-thick column densities, but high covering fractions $(40-90 \%)$ at $N_{\mathrm{H}}=10^{22} \mathrm{~cm}^{-2}$. These high covering fractions suggest that a substantial part of the type1/type 2 dichotomy is caused by galaxy-scale gas. This is consistent with the finding of Maiolino \& Rieke (1995) that nearby type2 AGN are often edge-on galaxies.

We note that our results tend to produce high covering fractions, which are only consistent with the measurements because of large uncertainties. Despite our efforts to mitig- ate stellar mass and metallicity biases, we suppose that the use of LGRB host galaxies in Paper I, may affect our results when applied to AGN host galaxies: LGRB host galaxies are actively star forming (Levesque 2014; Krühler 2015; Perley et al. 2016a) and thus may have slightly more gas than the average AGN galaxy. A correction in the column density by a factor of 2 would agree well with data points at all redshifts. This is demonstrated by the dashed line in Figure 1, which shows a normal distribution of column densities around $\log N_{\mathrm{H}} / \mathrm{cm}^{-2}=10^{22}$ with standard deviation 0.5 . With this potential systematic over-estimation in mind, a nuclear obscurer is then clearly needed at $N_{\mathrm{H}} \approx 10^{23.5} \mathrm{~cm}^{-2}$. In any case, our LGRB-based results are consistent with the current AGN surveys.

We evaluate the validity of using LGRBs to probe the galaxy obscuration of AGN below. Firstly, the stellar mass distributions of LGRBs and AGN are different. We incorporated this in Section 2 by deriving a stellar-mass dependent obscuration of LGRBs, and applied it to the measured stellar mass distribution of AGN. Secondly, LGRBs prefer galaxies with sub-solar stellar metallicities, so one may expect the gas inside them to be of lower metal content than AGN. Furthermore, the location of AGN in the metal-rich centre of galaxies may also increase their metal content, in contrast to LGRBs which explode in star-forming regions. When investigating these effects observationally in Paper I and here with simulations in Figure 3, we find that LOS metallicity is very comparable between the AGN and LGRB population. We speculate that this is because the LOS metallicity is averaged over long distances of the host galaxies. We emphasise that the simulations used here reproduce the observed hydrogen and CO masses in galaxies and simultaneously the galaxy stellar mass function. Thirdly, most $\mathrm{SMBH}$ accretion is thought to occur in galaxy-galaxy mergers (Sanders et al. 1988; Hopkins et al. 2006), although evidence of an elevated merger fraction in X-ray detected AGN is weak at high-redshift $(z>0.5)$ (Grogin et al. 2003, 2005; Pierce et al. 2007; Gabor et al. 2009; Kocevski et al. 
$2011,2015)$. It has therefore been suggested that only luminous AGN are preferentially associated with mergers (see e.g., Treister et al. 2012). Our LGRB-based results show that host galaxy obscuration is most important at the luminous end for Compton-thin obscuration. LGRB host galaxies may be appropriate in this comparison as they are also frequently merging or distorted systems (Fruchter et al. 2006; Wainwright et al. 2007). Finally, we investigated the obscuration of AGN by host-galaxy gas in the hydrodynamic simulations in Figure 2. The results are completely consistent with our GRB-based results: No Compton-thick columns are predicted for the AGN population, while substantial covering is found at $N_{\mathrm{H}} \approx 10^{22} \mathrm{~cm}^{-2}$. However, the obtained covering fractions depend on the feedback strength, and are distinctly different for the EAGLE and Illustris simulations; the latter implements strong feedback and results in lower covering fractions. Appendix A investigates this in more detail, and concludes that the fraction of obscured AGN can be used to rule out specific feedback implementations. From the aforementioned investigations, our approach of using LGRB hostgalaxy obscuration to constrain the galaxy-scale obscuration of AGN appears valid.

Our results give, for the first time, constraints on the galaxy-scale obscurer alone. We use this in the subsequent sections to disentangle the AGN nuclear obscurer from the galaxy-scale obscurer (Section 5.2), and to describe their behaviour as a function of accretion luminosity, black hole mass, host galaxy stellar mass and redshift (Section 6). Finally, physical effects leading to this behaviour are discussed in Section 6.2 .

\subsection{The nuclear obscurer is luminosity and mass-dependent}

We can now investigate the behaviour of the nuclear obscurer by subtracting the galaxy-scale obscuration. In particular, the observed luminosity-dependence of the obscurer is noteworthy. X-ray surveys consistently find a strong decline towards high luminosities (Ueda et al. 2003; Hasinger et al. 2005; La Franca et al. 2005; Ebrero et al. 2009; Ueda et al. 2014; Buchner et al. 2015; Aird et al. 2015) of the obscured fraction $N_{\mathrm{H}}>10^{22} \mathrm{~cm}^{-2}$ in the Compton-thin AGN population. The left panel of Figure 4 shows this decline from $\sim 70 \%$ to a persistent $\sim 40 \%$. According to our results, this can be interpreted as two scales contributing to the obscuration: a $40 \%$ galaxy-scale obscurer, and a luminositydependent nuclear obscurer. The former was constrained in this paper. The latter is the remainder still needed to fit the observations. According to this picture, the nuclear obscurer completely disappears toward high luminosities at $L(2-10 \mathrm{keV}) \approx 10^{44.5} \mathrm{erg} / \mathrm{s}$, and the only obscurer left is the host galaxy ${ }^{4}$.

The nuclear obscurer is however not only luminositydependent, but likely also black hole mass dependent. First evidence of this comes from noting that the luminositydependence is redshift-dependent, with higher redshifts having higher cut-off luminosities. This has been found by many works (Ueda et al. 2003; Ebrero et al. 2009; La Franca et al.

4 aside from the persistent, nuclear, Compton-thick obscurer
2005; Ueda et al. 2014; Aird et al. 2015) by fitting a empirical, parametric model to the relative number density derived from AGN surveys. Buchner et al. (2015) derived the same result using a non-parametric method, indicating that this change is indeed a robust result. In Buchner et al. (2015), the implications for obscurer models were discussed in their Section 5.3. As the luminosity dependence is observed to evolve over cosmic time, a black hole mass dependence needs to be invoked. They concluded that an Eddington-limited blow-out of the obscurer could explain the luminosity dependence, because the Eddington-limit is a function of luminosity and black hole mass. If the average, currently accreting black hole is more massive at high redshift than at low redshift, the turn-over luminosity decreases over cosmic time as observed. Such black hole mass downsizing also has evidence from optical observations of the black hole mass function evolution (Schulze \& Wisotzki 2010; Kelly \& Shen 2013; Schulze et al. 2015) and semianalytic models which reproduce the evolution of the AGN luminosity function (Fanidakis et al. 2012; Enoki et al. 2014; Hirschmann et al. 2014). Recently, Oh et al. (2015) found evidence that the type- 1 fraction in a SDSS selected sample is both luminosity and black hole mass dependent. Because of the aforementioned observations, simplistic models which only depend on luminosity such as the receding torus model (Lawrence 1991) have been ruled out.

We therefore need a luminosity and black hole mass dependent obscurer model to explain observations. Such an empirical model is presented in the next section.

\section{RADIATION-LIFTED TORUS - A NUCLEAR OBSCURER MODEL}

Because neither semi-analytic nor hydro-dynamic cosmological simulations resolve the nuclear obscurer of AGN, we present a sub-grid model for post-processing. We construct a model which reproduces the fraction of obscured AGN as a function of redshift and luminosity as discussed above. It can also serve as a summary of observational constraints when exploring physical models for the nuclear obscurer (see next section).

We assume that a nuclear Compton-thick obscurer covers a fraction of the SMBH, $f_{\mathrm{CT}} \sim 35 \%$. This fraction was measured directly from AGN surveys (Buchner et al. 2015), by matching the soft and hard X-ray luminosity functions (Aird et al. 2015) or by matching the Compton-thin X-ray luminosity function to the spectrum of the Cosmic X-ray background (Ueda et al. 2014). Similar fractions have recently been found in local surveys, e.g. Ricci et al. (2016).

We propose that the remaining Compton-thin sky is obscured by two components: galaxy-scale gas and a nuclear Compton-thin obscurer. We adopt the following formula for the covering fraction ${ }^{5}$ :

$$
f_{\text {cov }}=f_{\text {gal }}\left(M_{\star}\right)+f_{\text {nuc }} \cdot \exp \left\{-\frac{1}{2 \sigma^{2}}\left(\log \frac{L}{L_{\text {peak }}}\right)^{2}\right\}(6)
$$

The luminosity-independent obscuration, $f_{\text {gal }}$, is in general

${ }^{5}$ exceeding $N_{\mathrm{H}}=10^{22} \mathrm{~cm}^{-2}$ 

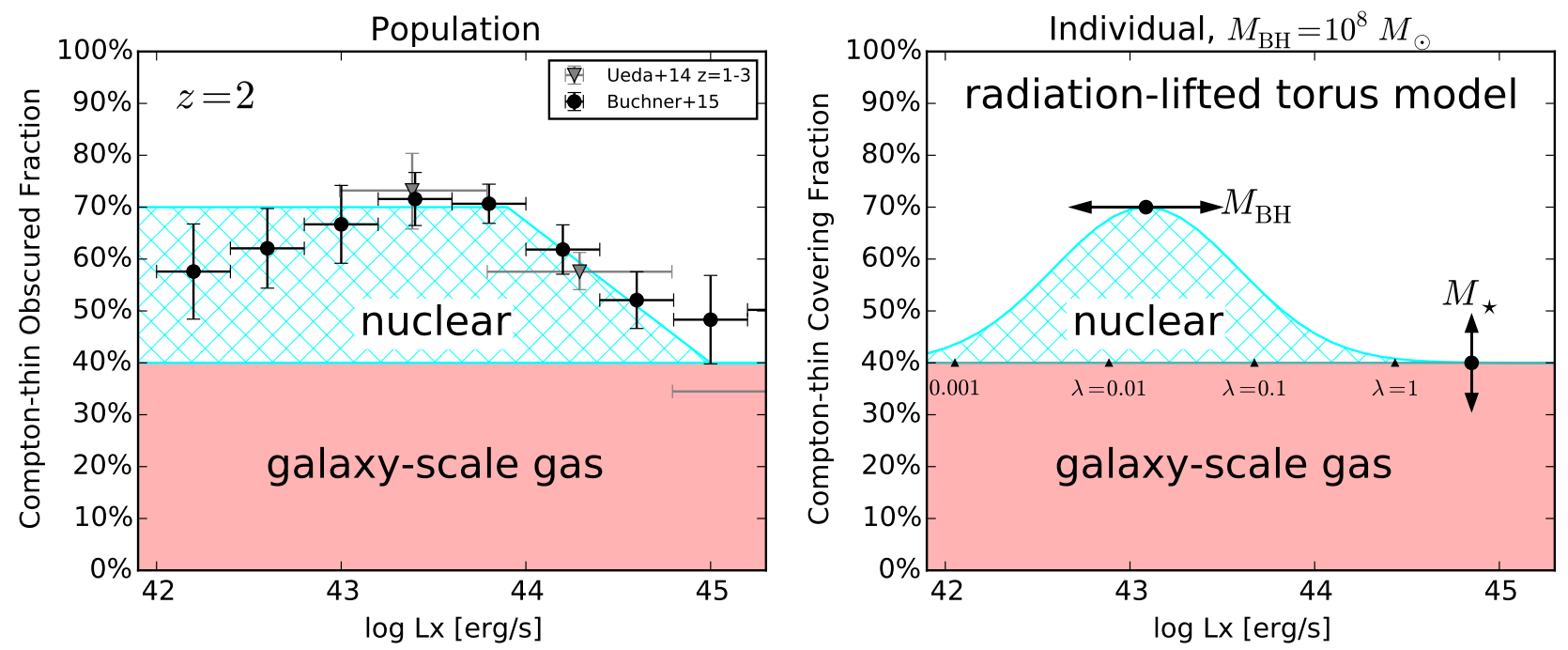

Figure 4. Left panel: The observed luminosity-dependence of the obscured fraction of Compton-thin AGN. With the galaxy-scale gas obscuration constrained and to first order independent of luminosity (red, see Section 3), we separate out the nuclear, variable obscurer (cyan, illustrative only) and develop a new model, the radiation-lifted torus model (Section 6). Right panel: In the radiation-lifted torus model, the nuclear obscuration of individual sources depends on accretion rate and black hole mass. Here the case of an individual black hole of mass $10^{8} M_{\odot}$ is shown. The nuclear obscuration (cyan) is largest at a few percent of the Eddington rate, and adds to the permanently present, stellar-mass dependent host galaxy-scale obscuration (red). Code in Listing 1.

Table 1. Parameters of the radiation-lifted torus model. The recommended values are constrained by local observations as described in Section 6. Example code can be found in Listing 1.

\begin{tabular}{ccc} 
Parameter & Symbol & Observed Range \\
\hline Compton-thick obscuration & $f_{\mathrm{CT}}$ & $20 \%-40 \%$ \\
Host galaxy obscuration & $f_{\text {gal }}$ & $0 \%-60 \%$ \\
Compton-thin nuclear obscuration & $f_{\text {nuc }}$ & $20 \%-40 \%$ \\
Luminosity-dependence width & $\sigma$ & $0-1$ \\
Reference mass & $M_{L 43}$ & $10^{7}-10^{8} M_{\odot}$ \\
Mass-dependence & $p$ & $0-2$ \\
Intrinsic luminosity $(2-10 \mathrm{keV})$ & $L$ & (dynamic)
\end{tabular}
$35 \%$ (fixed)
galaxy-dependent; $40 \%$ population average $30 \%$

0.5 $10^{7.87} M_{\odot}$

$2 / 3$ (consider also $0,1,4 / 3$ )

Determined from accretion rate using bolometric corrections of Marconi et al. (2004)

a function of host galaxy properties, but on average $\sim 40 \%$. In hydro-dynamic simulations the host galaxy obscuration it can be derived for individual galaxies through ray-tracing (see Section 4). Otherwise it can be calculated given a stellar mass using Equation 1.

The nuclear obscured fraction is luminosity-dependent. We chose a Gaussian form which requires fewer parameters than a linear decline (e.g. Ueda et al. 2014). The obscuration of Equation 6 reaches a average maximum of $\sim 70 \%$ at luminosity $L_{\text {peak }}$ (see Figure 4 ), and we therefore suggest $f_{\text {nuc }}=30 \%$. In other words, of the sky seen from the black hole $40 \%$ is covered by the host galaxy, and of the remainder the nuclear obscurer covers half. The obscured fraction declines towards both bright and faint ends to $f_{\text {gal }}$, with $\sigma$ giving the characteristic width of the transition. Evidence of the low-luminosity decline has been found in surveys of the local Universe (Burlon et al. 2011; Brightman \& Nandra 2011) and at high redshifts (Buchner et al. 2015).

The luminosity where the obscured fraction is highest, $L_{\text {peak }}$, is in turn a function of mass (see Section 5.1):

$L_{\text {peak }}=10^{43} \mathrm{erg} / \mathrm{s} \cdot\left(\frac{M_{\mathrm{BH}}}{M_{L 43}}\right)^{p}$
Here, at $M_{L 43}$ the distribution peaks at $L(2-10 \mathrm{keV})=$ $10^{43} \mathrm{erg} / \mathrm{s}$, which is $L_{\mathrm{bol}}=10^{10.7} L_{\odot}$ using the conversion of (Marconi et al. 2004). The black hole mass-dependence is defined by the $p$ parameter. At $p=0$, the radiationlifted torus is mass-independent, corresponding to a strict luminosity-dependent unified model. At $p=1$, it is only Eddington-rate dependent. The parameters $p, M_{L 43}$ and $\sigma$ are not known apriori. For mass-scaling, $p=2 / 3$ is suggested from the theoretical works on the obscurer by Elitzur \& Netzer (2016) and Wada (2015), however a wide range (e.g. $p=0-2$ ) may be considered (see also Hönig \& Beckert 2007). We derive fiducial values for the other two parameters using the Swift-BAT survey of local AGN. That survey reported a mean mass of $\log M_{\mathrm{BH}} / M_{\odot}=7.87$, a standard deviation of 0.66 dex and a skew towards low masses (Winter et al. 2009). Adopting an appropriate skewed normal distribution with skew parameter -10 (tail to low masses) around $M_{L 43}=10^{7.87} M_{\odot}$, we find that $\sigma \approx 0.5$ approximately reproduces the width of the obscured fraction function reported in Burlon et al. (2011), and peaks at $L_{\mathrm{X}}=10^{43} \mathrm{erg} / \mathrm{s}$.

Table 1 lists the parameters of the radiation-lifted torus model, with recommended typical values. We emphasise that the observations pertaining to the redshift evolution have 

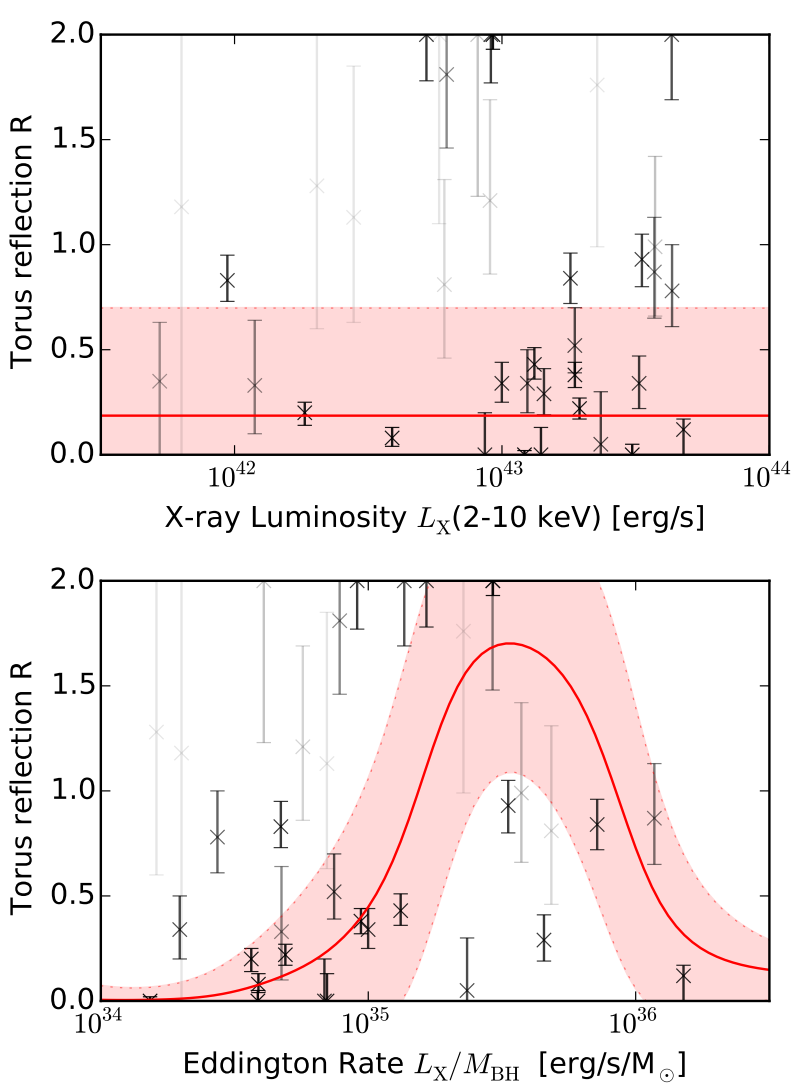

Figure 5. The Compton reflection strength, a proxy for the covering of the torus, as a function of accretion luminosity (top panel) and Eddington rate (bottom panel). Data points are taken from Kawamuro et al. (2016) and shaded proportional to the error size to highlight those with tighter constraints. Non-parametric data smoothing (red curve with $1 \sigma$ uncertainties as red shading) highlights that at intermediate Eddington rates the reflection strength appears elevated, while there is no clear trend with X-ray luminosity.

not been used when constructing our model. Cosmological simulations using the radiation-lifted torus model can thus compare be against those (e.g. Buchner et al. 2015). For simulations, Listing 1 shows Python code which generates column densities.

The right panel of Figure 4 illustrates the behaviour of the radiation-lifted torus model. The obscured fraction undergoes a luminosity and mass-dependent peak, where the Compton-thin medium is extended and occupies a substantial fraction of the sky. Owing to the host galaxy, a constant fraction is present at all luminosities and masses. The Compton-thick fraction here has been assumed to be constant, although we note that Ricci et al. (2016) claims a luminosity-dependence of the Compton-thick fraction.

\subsection{Predictions of the radiation-lifted torus model}

The radiation-lifted torus model predicts that the covering fraction of individual AGN depends to first order on Eddington rate, not luminosity alone. Kawamuro et al. (2016) recently analysed a sample of local $(z<0.1)$ Swift-BAT Compton-thin obscured AGN $\left(\log N_{\mathrm{H}}=10^{22-24} \mathrm{~cm}^{-2}\right)$ with broad-band Suzaku observations. They report that the Compton reflection component in the hard X-ray spectrum varies weakly with luminosity (their Figure 7 , left panel). They use Compton reflection as a proxy of the covering fraction and argue for a luminosity-dependent obscurer covering. We plot their data in the top panel of Figure 5. Overplotted we show a non-parametric fit using a Gaussian kernel density estimator. Instead of choosing bins manually, this procedure provides an optimal smoothing length by leaving one data point out in turn, predicting it from the remaining data, computing the prediction error and then decreasing the sum of these errors by varying the smoothing width. In the shown case, a wide kernel is optimal, which indicates that no strong trend is found, and the data scatters around a mean value without a strong trend. In other words, luminosity is not effective in predicting the reflection strength of individual AGN.

If we consider Eddington rate as the driver instead, we find a dependence, as shown in the lower panel of Figure 5. While there is substantial scatter in the data, the reflection strengths of well-constrained objects are high only for intermediate Eddington rates and low otherwise. The nonparametric fit (red curve) highlights this. Such a observed bulk increase is rarely expected, as randomly assigning Eddington rates shows $(p \approx 0.05)$. Therefore, Figure 5 demonstrates that the evidence for Eddington rate-dependent covering of individual AGN is stronger than for luminositydependent covering. We caution that the presented evidence is still relatively weak as the sample of Kawamuro et al. (2016) is heterogeneously selected and the Compton reflection strength $R$ is only a crude proxy for the covering of the obscurer. Upcoming Swift-BAT studies with detailed spectral analysis, completeness corrections and more black hole mass measurements (C. Ricci et al. in prep) will be in a better position to confirm or reject the radiation-lifted torus model.

The fraction of infrared reprocessed emission has been used to measure the covering factor of individual AGN. To first order, the obscurer covering fraction is the ratio of dust infrared re-radiated luminosity to bolometric illuminating luminosity. Surveys employing this method (e.g. Maiolino et al. 2007; Lusso et al. 2013) typically find fractions between $40 \%$ and $75 \%$ (luminosity-dependent). These fractions were corrected for anisotropic illumination and emission (from Stalevski et al. 2016, see their Figure 13). These measurements would include emission from the Compton-thick obscurer and the nuclear, Compton-thin obscurer. With our assumed fiducial values we obtain $35 \%\left(f_{\mathrm{CT}}\right)$ to $75 \%$ $\left(f_{\mathrm{CT}}+f_{\text {nuc }}\right)$ and therefore also agree with those observations. Infrared studies remain however difficult to use as a constraint, as the entire infrared SED has to be constrained for each object (Netzer et al. 2016) and covering factors have to be corrected based on uncertain model geometries (Stalevski et al. 2016).

\subsection{Physical obscurer processes}

Physical processes giving rise to the luminosity and massdependent behaviour cannot be discussed rigorously within the scope of this paper. However, we point out a few key results from recent theoretical works. Otherwise we refer to 


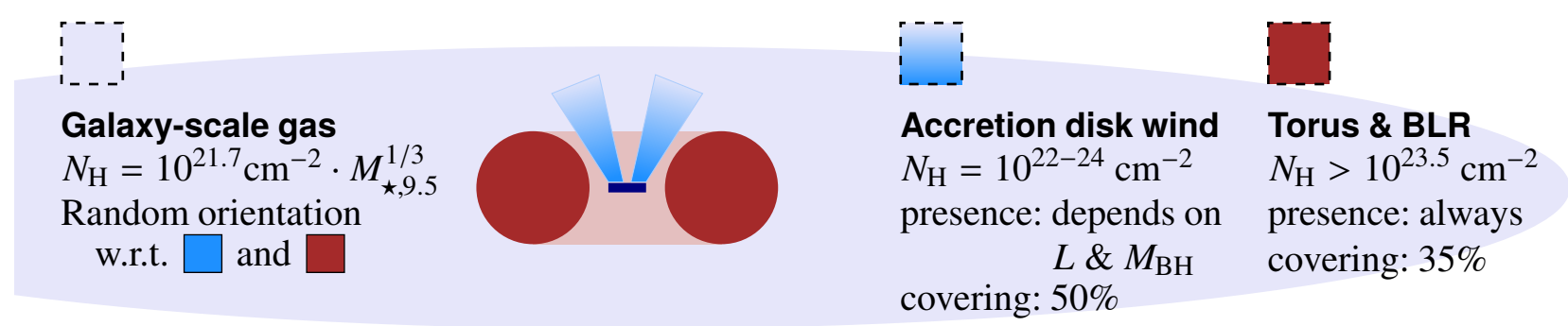

Figure 6. Cartoon of the three known obscurer components: Host galaxy obscuration (light blue), luminosity-dependent Compton-thin nuclear obscuration (blue) and Compton-thick nuclear obscuration (red). In this illustration, the Compton-thin obscurer is provided by a clumpy accretion disk wind, while the Compton-thick obscurer is a clumpy donut-like structure. Both are embedded in the host galaxy with random orientation. This is one physical scenario consistent with the proposed radiation-lifted torus model, other possibilities are outlined in Section 6.2.

the review of Hoenig (2013) which discusses current torus modelling approaches.

Strong unified obscurer models require that in every object an increase in the accretion luminosity anti-correlates with the extent of the obscurer. We take note of the analytic wind model formalism described in Elitzur \& Netzer (2016) and of the radiation-driven fountain model by Wada (2015). Both models produce a vertically extended obscurer structure as a function of luminosity and mass. At very low luminosities, radiation is not sufficient to puff up the obscurer. In the hydro-dynamical simulations of Wada (2012) very high accretion luminosities are associated with strong outflows, which suppress the vertical extent of the obscuring structure as they occupy larger angles. The cartoon of Figure 6 illustrates such an accretion disk wind scenario in combination with host galaxy obscuration and a Comptonthick torus for the three, distinct obscuring components. The opening angles shown are approximately correct. For visualisation purposes we have shown smooth gas distributions, while in reality the obscurer is thought to be clumpy (see e.g., Markowitz et al. 2014, and references therein). To date, models of the nuclear obscurer largely lack observational constraints. Our radiation-lifted torus model summarises observational boundary constraints of covering fractions and column densities for how a physical "torus" model should behave.

Sazonov et al. (2015) invoked anisotropic X-ray emission to explain the luminosity-dependence of the obscurer. If the X-ray emission is more luminous towards the lessobscured poles while less luminous towards the more obscured plane, in a coordinate system defined by the accretion disk. They argue that such anistropy is expected from accretion disks. Inverse Compton scattering of UV accretion disk photons in a hot electron plasma near the accretion disk is thought to produce the X-ray spectrum of AGN (Katz 1976; Sunyaev \& Titarchuk 1980, 1985). Because several such scatterings are needed, it is unclear if the anistropy would remain (depends on the plasma geometry, see e.g. Yao et al. 2005). Anisotropic X-ray emission would also predict that AGN with detected maser disks, which are believed to be viewed edge-on, should have higher observed Eddington ratios, and thus steeper X-ray spectral slopes, while this is not observed (Brightman et al. 2016). We also note that the scenario proposed by Sazonov et al. (2015) naturally predicts that the luminosity-dependence of the obscuration should evolve over time, in lock-step with the evolution of the knee of the luminosity function. Overall, this scenario deserves further study and can be formally treated as another model of the nuclear obscurer.

A further, commonly overlooked aspect is the triggering mechanism of AGN. Faint AGN are much more common than luminous AGN (e.g. Barger \& Cowie 2005; Ueda et al. 2003; Aird et al. 2010), a fact that needs to be explained with the triggering and light-curve of accretion events. Galaxygalaxy mergers are today thought to be the main trigger of luminous AGN activity and efficient SMBH growth. This is because SMBHs need to accrete substantial fractions of their host galaxy gas (as shown by scaling relations) within durations comparable to the dynamical timescales of galaxy centres (Somerville et al. 2008). In the time-line proposed by Hopkins et al, the luminous phase occurs in relatively late merger phases. Luminous AGN stop further infall by radiation pressure and quickly reduce their column densities (Hopkins et al. 2006). In contrast, the faint AGN population is suggested to be dominated by periods before and after peak accretion (Hopkins et al. 2005b). Early merger phases may have enhanced obscuration (Hopkins et al. 2005a, 2006), both galaxy-scale (Compton-thin) and nuclear (Compton-thin and Compton-thick). Additionally, a substantial part of the faint AGN population is probably associated with secular triggering mechanisms (e.g. Treister et al. 2012). Based on these considerations, one may therefore argue for an evolutionary SMBH "life" consisting of [illustrative time share in brackets]: (1) no accretion, and therefore no AGN detection [89\%], (2) nearby gas leading to obscuration and triggering of a faint AGN [10\%], either at the onset of a merger or through secular accretion events, (3) major merger triggering a bright AGN, which immediately clears the vertically extended obscurer but shines for a while before fading [1\%]. Such a duty cycle would give rise to the observed obscured fractions (Figure 4) while also respecting the luminosity function of AGN. To summarise, luminous AGN and faint AGN may live in different environments with different gas reservoirs feeding them; therefore unifying the obscuration properties in a luminosity-dependent torus may not be appropriate. 


\section{CONCLUSIONS}

Using only observational relations, we predict the covering fractions of galaxy-scale gas as relevant for the AGN population.

Our findings can be summarised as follows:

(i) Galaxy-scale gas does not provide Compton-thick lines of sight. We therefore conclude that heavily obscured AGN are associated with nuclear obscuration, and propose the value $N_{\mathrm{H}}=10^{23.5} \mathrm{~cm}^{-2}$ as a demarcation line for singling out the nuclear obscurer.

(ii) Galaxy-scale gas covers substantial fractions of the SMBH population at $N_{\mathrm{H}} \approx 10^{22-23.5} \mathrm{~cm}^{-2}$, sufficient to fully explain the obscured fraction at the bright end.

(iii) We constrain the nuclear obscurer by subtracting the galaxy-scale obscuration. A Compton-thick covering of $35 \%$ is necessary. The extent of the Compton-thin obscurer depends on both accretion luminosity and black hole mass.

(iv) We present the empirical radiation-lifted torus obscurer model (Section 6). It describes the behaviour of the AGN nuclear obscurer (torus) and galaxy-scale obscurer as a function of accretion luminosity, black hole mass, host galaxy stellar mass and is consistent with the available observations. We discuss physical effects which may give rise to this behaviour. This model can be used as a sub-grid model to post-process cosmological simulations.

(v) We investigated the inside of simulated galaxies from state-of-the-art hydro-dynamic, cosmological simulations. These verified the assumptions in our approach by raytracing the galaxy gas. Some of these simulations produce obscured fractions consistent with observations. However, the results are highly sensitive to the adopted feedback models. We therefore suggest the Compton-thin obscured AGN fraction as a diagnostic to rule out feedback models. This diagnostic can be applied already at early cosmic times $(z=2-3)$.

\section{ACKNOWLEDGEMENTS}

We thank the anonymous referee for useful comments. JB thanks Antonis Georgakakis and Dave Alexander for insightful conversations. JB thanks Joop Schaye, Matthieu Schaller and Yetli Rosas-Guevara for detailed comments on the analysis of hydrodynamic simulations. JB thanks Klaus Dolag, Sergio Contreras and Torsten Naab for conversations about hydro-dynamic simulations.

We acknowledge support from the CONICYT-Chile grants Basal-CATA PFB-06/2007 (JB, FEB), FONDECYT Regular 1141218 (FEB), FONDECYT Postdoctorados 3160439 (JB), "EMBIGGEN" Anillo ACT1101 (FEB), and the Ministry of Economy, Development, and Tourism's Millennium Science Initiative through grant IC120009, awarded to The Millennium Institute of Astrophysics, MAS (JB, FEB).

\section{References}

Aird J., et al., 2010, MNRAS, 401, 2531

Aird J., et al., 2012, ApJ, 746, 90

Aird J., et al., 2013, ApJ, 775, 41 $\overline{\text { Algorithm } 1 \text { Python code for generating a column density }}$ under the radiation-lifted torus model. Returns for a specific AGN with a given X-ray luminosity and black hole mass either a unobscured, Compton-thin obscured or Comptonthick obscured column. The parameters are described in Section 6 and listed in Table 1. The relations of Marconi et al. (2004) are useful to convert bolometric accretion luminosities to intrinsic $2-10 \mathrm{keV}$ X-ray luminosity.

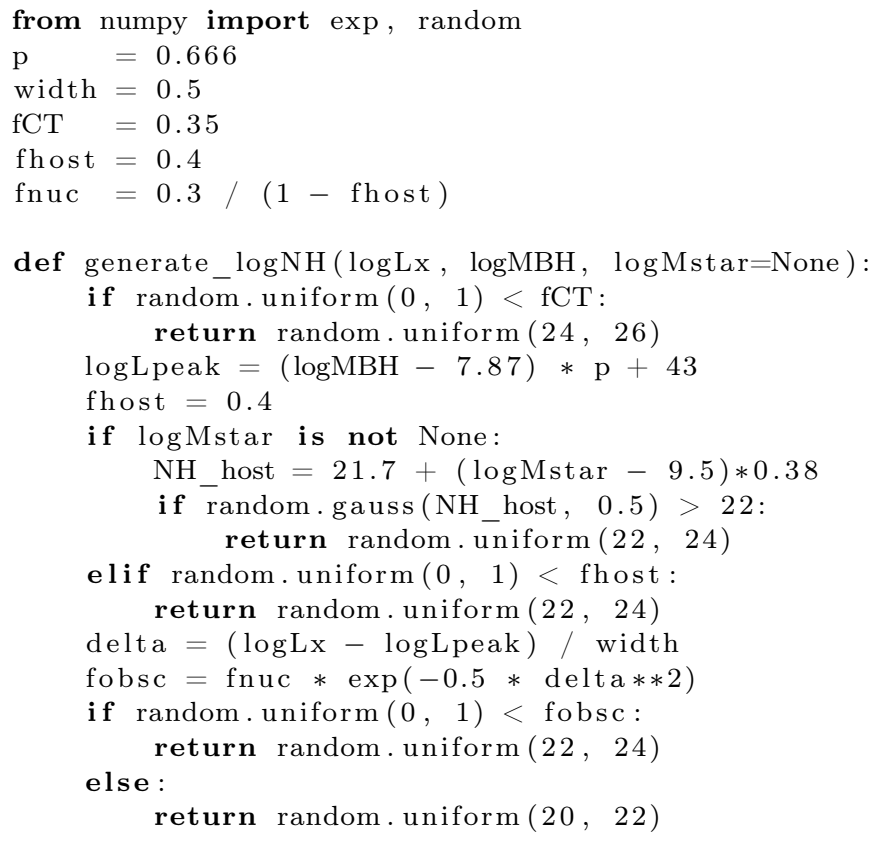

Aird J., Coil A. L., Georgakakis A., Nandra K., Barro G., PerezGonzalez P. G., 2015, preprint, (arXiv:1503.01120)

Bahe Y. M., et al., 2015, preprint, (arXiv:1511.04909)

Barger A. J., Cowie L. L., 2005, ApJ, 635, 115

Bloom J. S., Kulkarni S. R., Djorgovski S. G., 2002, AJ, 123, 1111

Bongiorno A., et al., 2012, MNRAS, 427, 3103

Brightman M., Nandra K., 2011, MNRAS, 414, 3084

Brightman M., Nandra K., Salvato M., Hsu L.-T., Aird J., Rangel C., 2014, MNRAS, 443, 1999

Brightman M., et al., 2016, preprint, (arXiv:1606.09265)

Buchner J., et al., 2015, ApJ, 802, 89

Burlon D., Ajello M., Greiner J., Comastri A., Merloni A., Gehrels N., 2011, ApJ, 728, 58

Cox D. P., 2005, ARA\&A, 43, 337

Crain R. A., et al., 2015, MNRAS, 450, 1937

Croton D. J., et al., 2006, MNRAS, 365, 11

Croton D. J., et al., 2016, preprint, (arXiv: 1601.04709)

Cui W., Power C., 2016, MNRAS, 458, 4052

Dickey J. M., Lockman F. J., 1990, ARA\&A, 28, 215

Ebrero J., et al., 2009, A\&A, 493, 55

Elitzur M., 2006, New Astron. Rev., 50, 728

Elitzur M., Netzer H., 2016, preprint, (arXiv:1603.04909)

Enoki M., Ishiyama T., Kobayashi M. A. R., Nagashima M., 2014, ApJ, 794, 69

Fanidakis N., et al., 2012, MNRAS, 419, 2797

Fruchter A. S., et al., 2006, Nature, 441, 463

Furlong M., et al., 2015a, preprint, (arXiv:1510.05645)

Furlong M., et al., 2015b, MNRAS, 450, 4486

Gabor J. M., et al., 2009, ApJ, 691, 705

Genel S., et al., 2014, MNRAS, 445, 175

Goulding A. D., Alexander D. M., 2009, MNRAS, 398, 1165

Grogin N. A., et al., 2003, ApJ, 595, 685 
Grogin N. A., et al., 2005, ApJ, 627, L97

Hasinger G., Miyaji T., Schmidt M., 2005, A\&A, 441, 417

Hirschmann M., Naab T., Somerville R. S., Burkert A., Oser L., 2012, MNRAS, 419, 3200

Hirschmann M., Dolag K., Saro A., Bachmann L., Borgani S., Burkert A., 2014, MNRAS, 442, 2304

Hjorth J., Bloom J. S., 2012, The Gamma-Ray Burst - Supernova Connection. pp 169-190, http://arxiv.org/pdf/1104. $2274 \mathrm{v} 1$

Hoenig S. F., 2013, preprint, (arXiv:1301.1349)

Hönig S. F., Beckert T., 2007, MNRAS, 380, 1172

Hopkins P. F., Hernquist L., Cox T. J., Di Matteo T., Martini P., Robertson B., Springel V., 2005a, ApJ, 630, 705

Hopkins P. F., Hernquist L., Cox T. J., Di Matteo T., Robertson B., Springel V., 2005b, ApJ, 630, 716

Hopkins P. F., Hernquist L., Cox T. J., Di Matteo T., Robertson B., Springel V., 2006, ApJS, 163, 1

Ilbert O., et al., 2013, A\&A, 556, A55

Kalberla P. M. W., Burton W. B., Hartmann D., Arnal E. M., Bajaja E., Morras R., Pöppel W. G. L., 2005, A\&A, 440, 775

Katz J. I., 1976, ApJ, 206, 910

Kawamuro T., Ueda Y., Tazaki F., Ricci C., Terashima Y., 2016, preprint, (arXiv:1606.04941)

Kelly B. C., Shen Y., 2013, ApJ, 764, 45

King A. R., Pringle J. E., 2006, MNRAS, 373, L90

Kocevski D. D., et al., 2011, preprint, (arXiv:1109.2588)

Kocevski D. D., et al., 2012, ApJ, 744, 148

Kocevski D. D., et al., 2015, ApJ, 814, 104

Krolik J. H., Begelman M. C., 1988, ApJ, 329, 702

Krühler T., 2015, Astronomische Nachrichten, 336, 487

La Franca F., et al., 2005, ApJ, 635, 864

Lagos C. d. P., et al., 2015, MNRAS, 452, 3815

Lawrence A., 1991, MNRAS, 252, 586

Levesque E. M., 2014, PASP, 126, 1

Lu Y., Mo H. J., Lu Z., 2015, preprint, (arXiv:1504.02109)

Lusso E., et al., 2013, ApJ, 777, 86

Maiolino R., Rieke G. H., 1995, ApJ, 454, 95

Maiolino R., Shemmer O., Imanishi M., Netzer H., Oliva E., Lutz D., Sturm E., 2007, A\&A, 468, 979

Marconi A., Risaliti G., Gilli R., Hunt L. K., Maiolino R., Salvati M., 2004, MNRAS, 351, 169

Markowitz A. G., Krumpe M., Nikutta R., 2014, MNRAS, 439, 1403

Matt G., 1997, Mem. Soc. Astron. Italiana, 68, 127

Matt G., 2000, A\&A, 355, L31

Molinari S., et al., 2011, ApJ, 735, L33

Morris M., Serabyn E., 1996, ARA\&A, 34, 645

Mullaney J. R., et al., 2015, MNRAS, 453, L83

Muzzin A., et al., 2013, ApJ, 777, 18

Nelson D., Vogelsberger M., Genel S., Sijacki D., Kereš D., Springel V., Hernquist L., 2013, MNRAS, 429, 3353

Netzer H., Lani C., Nordon R., Trakhtenbrot B., Lira P., Shemmer O., 2016, ApJ, 819, 123

Oh K., Yi S. K., Schawinski K., Koss M., Trakhtenbrot B., Soto K., 2015, ApJS, 219, 1

Perley D. A., Niino Y., Tanvir N. R., Vergani S. D., Fynbo J. P. U., 2016a, Space Sci. Rev.,

Perley D. A., et al., 2016b, ApJ, 817, 7

Perley D. A., et al., 2016c, ApJ, 817, 8

Pierce C. M., et al., 2007, ApJ, 660, L19

Pontzen A., et al., 2010, MNRAS, 402, 1523

Prieto M. A., Mezcua M., Fernández-Ontiveros J. A., Schartmann M., 2014, MNRAS, 442, 2145

Ricci C., Ueda Y., Koss M. J., Trakhtenbrot B., Bauer F. E., Gandhi P., 2016, preprint, (arXiv:1603.04852)

Risaliti G., Maiolino R., Salvati M., 1999, ApJ, 522, 157

Rosario D. J., et al., 2011, preprint, (arXiv:1110.3816)

Rosario D. J., et al., 2013, ApJ, 771, 63
Rosas-Guevara Y., Bower G. R., Schaye J., McAlpine S., DallaVecchia C., Frenk S. C., Schaller M., Theuns T., 2016, preprint, (arXiv:1604.00020)

Sanders D. B., Soifer B. T., Elias J. H., Madore B. F., Matthews K., Neugebauer G., Scoville N. Z., 1988, ApJ, 325, 74

Santini P., et al., 2012, A\&A, 540, A109

Sazonov S., Churazov E., Krivonos R., 2015, preprint, (arXiv: 1509.01259)

Scannapieco C., et al., 2012, MNRAS, 423, 1726

Schaller M., Dalla Vecchia C., Schaye J., Bower R. G., Theuns T., Crain R. A., Furlong M., McCarthy I. G., 2015, MNRAS, 454, 2277

Schaye J., et al., 2015, MNRAS, 446, 521

Schulze A., Wisotzki L., 2010, A\&A, 516, A87

Schulze A., et al., 2015, MNRAS, 447, 2085

Sembolini F., et al., 2016, MNRAS,

Sijacki D., Vogelsberger M., Genel S., Springel V., Torrey P., Snyder G. F., Nelson D., Hernquist L., 2015, MNRAS, 452, 575

Somerville R. S., Hopkins P. F., Cox T. J., Robertson B. E., Hernquist L., 2008, MNRAS, 391, 481

Stalevski M., Ricci C., Ueda Y., Lira P., Fritz J., Baes M., 2016, MNRAS,

Sunyaev R. A., Titarchuk L. G., 1980, A\&A, 86, 121

Sunyaev R. A., Titarchuk L. G., 1985, A\&A, 143, 374

Tacconi L. J., et al., 2013, ApJ, 768, 74

Treister E., et al., 2004, ApJ, 616, 123

Treister E., Schawinski K., Urry C. M., Simmons B. D., 2012, ApJ, 758, L39

Ueda Y., Akiyama M., Ohta K., Miyaji T., 2003, ApJ, 598, 886

Ueda Y., Akiyama M., Hasinger G., Miyaji T., Watson M. G., 2014, ApJ, 786, 104

Vergani S. D., et al., 2015, A\&A, 581, A102

Vogelsberger M., Sijacki D., Kereš D., Springel V., Hernquist L., 2012, MNRAS, 425, 3024

Vogelsberger M., et al., 2014a, MNRAS, 444, 1518

Vogelsberger M., et al., 2014b, Nature, 509, 177

Wada K., 2012, ApJ, 758, 66

Wada K., 2015, preprint, (arXiv:1509.00576)

Wainwright C., Berger E., Penprase B. E., 2007, ApJ, 657, 367

Wilms J., Allen A., McCray R., 2000, ApJ, 542, 914

Winter L. M., Mushotzky R. F., Reynolds C. S., Tueller J., 2009, ApJ, 690, 1322

Yao Y., Zhang S. N., Zhang X., Feng Y., Robinson C. R., 2005, ApJ, 619, 446

\section{APPENDIX A: THE IMPACT OF SUB-GRID PHYSICS ON SIMULATED COVERING FRACTIONS}

We discuss three aspects which affect the results: (1) different sub-grid physics, most notably stronger feedback mechanisms, (2) differences between active and passive galaxies, (3) unresolved substructure of the gas.

The strength of EAGLE is that we can explore how variations of the physics affect the results. We focus on the fraction of AGN with $N_{\mathrm{H}}>10^{22} \mathrm{~cm}^{-2}$, and compare with EAGLE physics variations in the top panel of Figure A1. Recent large-scale AGN surveys (e.g. Ueda et al. 2014; Buchner et al. 2015) find a intrinsic fraction of about $\sim 40 \%$, with uncertainties encompassing the region $25-65 \%$ (dotted vertical lines). We consider any simulation with fractions outside as ruled out by observations. At $z=1$ (crosses), this nicely separates the EAGLE physics variations into two groups, one close to the observational constraints, and 

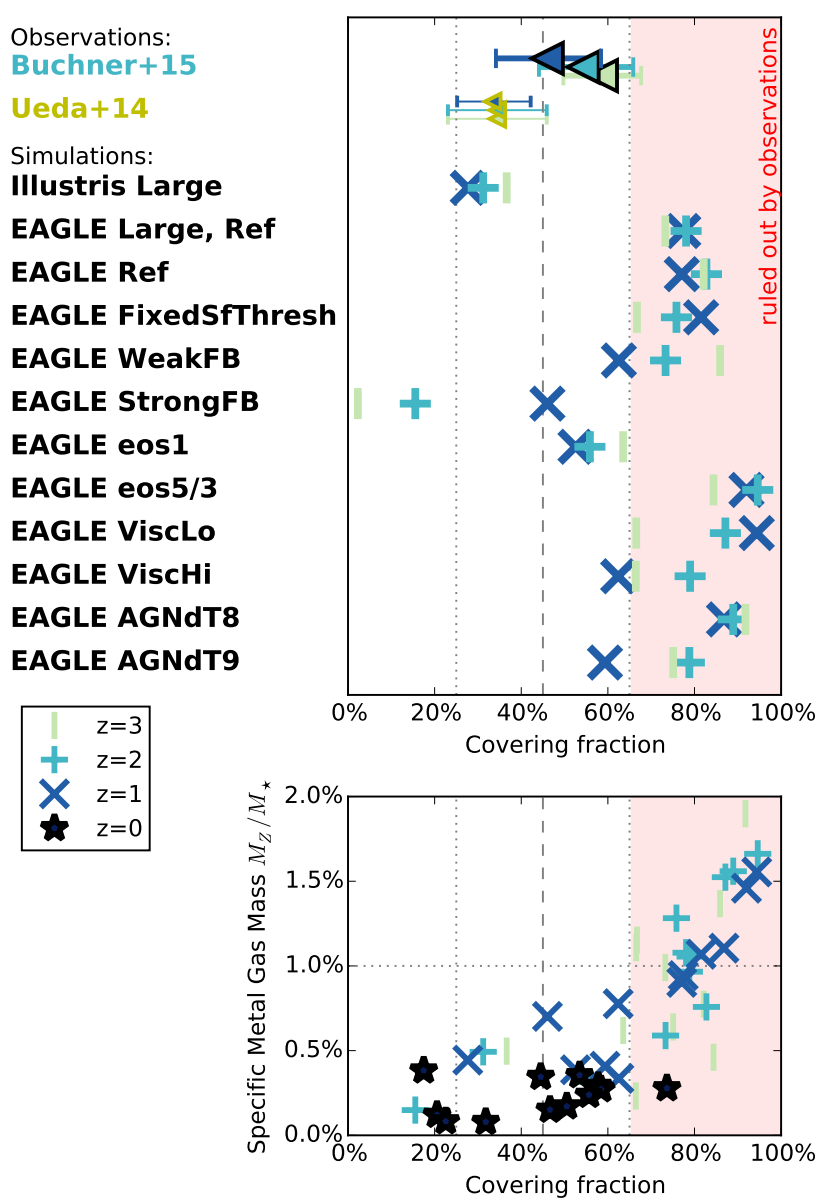

Figure A1. Obscured fractions from various simulations. Top panel: We compare the fraction of obscured, Compton-thin AGN at $z=1-3$ from observations (left-pointing triangles) to various cosmological simulations. Vertical lines indicate covering fractions of $25 \%$ (dotted), $45 \%$ (dashed) and $65 \%$ (dotted). Only some simulations consistently fall within the dotted lines. Bottom panel: The obscured fraction correlates with the average metal gas fraction of galaxies. Only simulations with specific metal gas fractions of $M_{\mathrm{Z}} / M_{\star}<1 \%$ yield covering fractions consistent with observations.

one significantly over-predicting the fraction of obscured AGN. The EAGLE reference simulation in a large cosmological volume (L0100N1504_REFERENCE) belongs to the latter group, as do the simulation runs in medium-size volumes (L0025N0752_REFERENCE, L0025N0752_RECALIBRATED). We now investigate which changes make the simulation agree better. Crain et al. (2015) presents these variations in detail.

Star formation-related feedback (supernovae, stellar winds, radiation pressure, cosmic rays) was altered in the WeakFB and StrongFB models. The efficiency threshold was modified by factors of 0.5 and 2 respectively, relative to the reference model. Here we find, surprisingly, that both models produce lower obscured fractions than the reference model. Strong feedback leads to underproduction of massive galaxies at $M_{\star}>10^{10.5} M_{\odot}($ Crain et al. 2015, their Figure 10), thereby biasing the galaxy population to small gas masses. It is less clear why the WeakFB produces a small obscured fraction. Presumably the feedback is not sufficient to ver- tically puff up galaxies, thereby reducing covering fractions. However, these variations are ruled out by other observations, e.g. the SMF (Crain et al. 2015, their Figure 10).

Next we discuss the effects of feedback from accreting SMBHs. In the EAGLE simulation, three parameters affect the triggering, efficiency and impact of AGN feedback respectively. The equation of state of the ISM can be modified from its reference value $(4 / 3)$ to 1 (eos 1 ). The increased sound speed then increases the accretion onto black holes in the simulation, which increases AGN feedback. Once near the black hole, matter is placed into the black hole with a Bondi accretion formula modified by a viscosity parameter. Increasing the viscosity (ViscHi) allows gas to loose angular momentum and accrete more efficiently. Once accreted, the temperature of particles near AGN is increased by $\Delta T=10^{8.5} \mathrm{~K}$, stochastically, bringing the gas into the metal cooling regime. Schaye et al. (2015) test the impact of increasing this temperature to $\Delta T=10^{9} \mathrm{~K}$ (AGNdT9). Each of these three modifications (eos1, ViscHi, AGNdT9) leads to a reduction of the obscured fraction of AGN (see Figure A1), while contrary modifications (eos5/3, ViscLo, AGNdT8) do the opposite. It is worth noting that Schaye et al. (2015) preferred the AGNdT9 variation over the reference simulation because it better fits soft X-ray observations of cluster gas. We also point out that Illustris uses relatively strong feedback, as it implements three different AGN feedback schemes (thermal, kinetic and radiation), whereas EAGLE uses only stochastic heating.

AGN outflows or radiation pressure may decrease the covering fractions momentarily. We have so far considered all simulated galaxies and found that they produce high covering fractions. Arguably these fractions are consistent with the covering fraction of low-luminosity AGN. Therefore, one could propose that AGN feedback at high luminosities modifies the galaxy in such way that covering fractions are reduced. Apriori this proposal appears unlikely, because nuclear gas should be affected first. Additionally, studies comparing the morphology of active and passive galaxies have found little evidence that these are different, by comparing appearances with asymmetry and concentration measures (Grogin et al. 2003, 2005; Pierce et al. 2007; Gabor et al. 2009; Kocevski et al. 2012) or visual classification (Kocevski et al. 2012). Indeed, our results remain unchanged if in the EAGLE reference simulation we only consider simulated galaxies with instantaneous black hole accretion rates corresponding to $L(2-10 \mathrm{keV})>10^{42} \mathrm{erg} / \mathrm{s}$, assuming a radiative efficiency of $10 \%$ and bolometric corrections of Marconi et al. (2004). In fact, because active galaxies are preferentially gas-rich, star-forming galaxies in that simulation, the average column density is higher by a factor of 2 , which increases the discrepancy.

A clumpy ISM may decrease the covering fractions. For instance the galactic ISM is structured into parsec-size clumps with filling factors of $1 \%$ (Cox 2005). Such clumps could not be resolved by simulations. However, as a LOS passes through large distances of the ISM ( 1 - few kiloparsecs), this clumpiness averages out. Additionally, clumpiness would effectively only redistribute the obscured fraction to both lower and higher column densities, potentially violating the constraints of higher column densities. There are also differences in the hydrodynamics code schemes and their accuracy. However, these are less important than the 
chosen sub-grid models (J. Shaye, priv. comm., see Scannapieco et al. 2012; Schaller et al. 2015; Cui \& Power 2016; Sembolini et al. 2016) in the present non-classical SPH simulations.

To summarise, our obscured fraction diagnostic is a highly sensitive test of feedback recipes. It can easily rule out feedback models already at early times (e.g. $z=2-3$ ) in the simulations, if they produce very high fractions of obscured AGN. This effectively places limits on the metal gas mass content of galaxies at $z=0-3$, as the bottom panel of Figure A1 demonstrates. There we plot the average specific metal gas mass $M_{\mathrm{Z}} / M_{\star}$ that is bound to the galaxy and within $10 \mathrm{kpc}$. The average was computed by fitting a powerlaw at $M_{\star}>10^{9.5} M_{\odot}$ with $3 \sigma$ clipping, and evaluating $M_{\mathrm{Z}} / M_{\star}$ at $M_{\star}=10^{10} M_{\odot}$. Adopting a moving average median instead gives comparable results. We find that the specific metal gas mass correlates well with the obscured fraction. The fact that unobscured AGN are found, i.e. $f_{\text {obsc }} \leq 65 \%$, implies that for the present set of simulations we can place a limit on the specific metal gas mass of $M_{\mathrm{Z}}<1 \% \cdot M_{\star}$, for the redshift range $z=0-3$.

\section{APPENDIX B: THE GAS MASS INSIDE GALAXIES}

Important constraints on how much gas resides in galaxies can be drawn from cosmological simulations. Such simulations evolve the matter density available at the Big Bang into collapsing bound structures. Semi-analytic models successfully reproduce many features of galaxies, including the stellar mass function of galaxies and their colour distribution (Croton et al. 2006; Somerville et al. 2008; Hirschmann et al. 2012; Fanidakis et al. 2012). As an illustrative case, we consider the model of Croton et al. (2016). Figure B1 plots the metal gas mass (the X-ray obscurer) present as a function of galaxy stellar mass. The median (red curve) falls consistently in the 1:30 to 1:100 range for the ratio of metal gas mass to stellar mass. With AGN host galaxies primarily drawn around the $M_{\star}=10^{10-11} M_{\odot}$ regime, the total gas available to obscure a central point source is about $M_{\mathrm{Z}} \sim 10^{9} M_{\odot}$. The Illustris simulation shows very similar results at $z=1-3$ obeying the same gas ratios. However at $z=0$, the gas content at the high-mass end is reduced because of the strong feedback implemented in that simulation.

We present a simple calculation to show that Comptonthick column densities (i.e. $N_{\mathrm{H}}>1.5 \times 10^{24} \mathrm{~cm}^{-2}$ ) cannot be achieved by accumulating the galaxy gas over several kpc. In X-ray spectral analysis, the equivalent hydrogen column density $N_{\mathrm{H}}$ is usually computed assuming solar abundances. To mimic this, we convert the metal mass in particles to the number of hydrogen atoms assuming solar mass fractions of the nearby ISM from Wilms et al. (2000):

$$
n_{\mathrm{H}}=\left.\frac{f_{\mathrm{X}}}{f_{\mathrm{Z}}}\right|_{\text {solar }} \times \frac{\rho_{\mathrm{Z}}}{m_{\mathrm{H}}}
$$

Inserting numbers including the hydrogen mass $m_{\mathrm{H}}$ we find

$$
n_{\mathrm{H}}=0.737 \times 10^{22} \mathrm{~cm}^{-2} \frac{1}{1 \mathrm{kpc}} \times \frac{\rho_{\mathrm{Z}}}{10^{6} M_{\odot} \mathrm{kpc}^{-3}} .
$$

For example, a $1 \mathrm{kpc}$ ray in a region of metal gas density
$10^{6} M_{\odot} / \mathrm{kpc}^{3}$ results in a measured column density of $N_{\mathrm{H}} \approx$ $10^{22} \mathrm{~cm}^{-2}$.

The gas inside a galaxy may be arranged in a multitude of ways to achieve a covering with column density $N_{\mathrm{H}}$. If we consider only gas outside a certain radius $R$, the most effective obscurer, i.e. the one with the least mass but complete covering, is an infinitely thin shell at that radius $R$. Its mass is easily computed as

$M_{\mathrm{H}}\left(N_{\mathrm{H}},>R\right)=4 \pi \cdot R^{2} \times N_{\mathrm{H}} \times m_{\mathrm{H}}$.

Converting to metals using the factor $\left.\frac{f_{\mathrm{Z}}}{f_{\mathrm{X}}}\right|_{\text {solar }}$ and expressing in conventional units, this limit is

$M_{\mathrm{Z}}\left(N_{\mathrm{H}},>R\right)=2.6 \times 10^{9} M_{\odot} \cdot \frac{N_{\mathrm{H}}}{1.5 \times 10^{24} \mathrm{~cm}^{-2}} \cdot\left(\frac{R}{1 \mathrm{kpc}}\right)^{2}$.

Therefore, a metal mass larger than $2.6 \times 10^{9} M_{\odot}$ is required to create a Compton-thick obscurer outside the central $1 \mathrm{kpc}$. Or equivalently, a metal mass of $2.6 \times 10^{9} M_{\odot}$ has to be brought to the central kpc to act as a Compton-thick obscurer. Note that this mass limit scales with the covering factor; for example obscuration of $10 \%$ of the sky requires $10 \%$ of the mass. This simple limit is shown for several levels of obscuration in Figure B2. Risaliti et al. (1999) already noted that such large masses at radii further than a few 10pc are ruled out in NGC1068 and Circinus, because they would gravitationally dominate the central region.

Given that metal gas masses are below $2.6 \times 10^{9} M_{\odot}$ in all but the most extreme galaxies (Figure B1), it follows that galaxy gas, when outside the central $1 \mathrm{kpc}$, is incapable of Compton-thick obscuration with substantial covering angles. In other words, some of that mass has to reside inside the central $1 \mathrm{kpc}$ to create Compton-thick covering. Admittedly, this is a weak constraint. However the result holds independently of the geometry of the gas, the galaxy type and is also applicable to mergers. As an example, we consider a $M_{\star}=10^{9} M_{\odot}$ galaxy merging into a $M_{\star}=10^{10} M_{\odot}$ galaxy (minor merger). Furthermore, lets assume that all of its gas $\left(M_{Z} \approx 10^{7} M_{\odot}\right)$ is made available as an obscurer. From Figure B2, we infer that the entire amount of gas must land within 100pc of the AGN in order to completely enshroud the SMBH in Compton-thick columns. More quantitative conclusions depend on the geometrical distribution of the gas in the galaxy. We analyse the galaxies produced by hydrodynamic simulations in Section 4. 


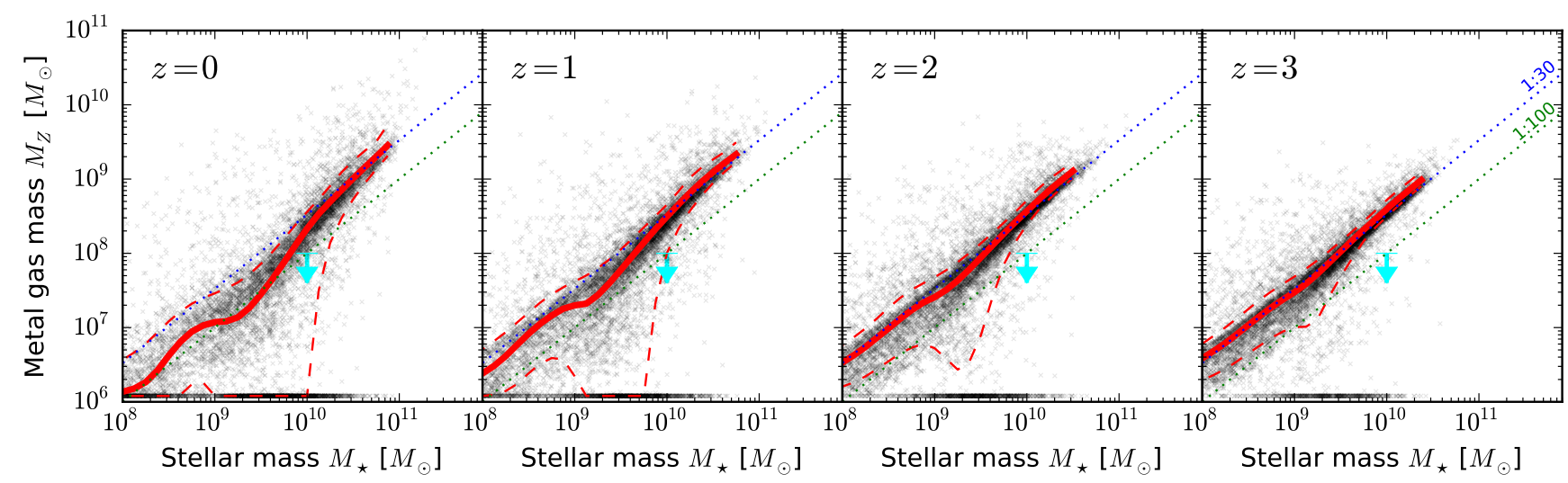

Figure B1. The metal gas mass in galaxies according to the SAGE semi-analytic model. The solid red curve indicates the median, while dashed red curves are the $1 \sigma$-equivalent quantiles. The ratio of metal gas to stellar mass lies between $1: 30$ and $1: 100$ (dotted lines). The magenta upper limit is derived in Appendix A for the mass of the central $10 \mathrm{kpc}$.

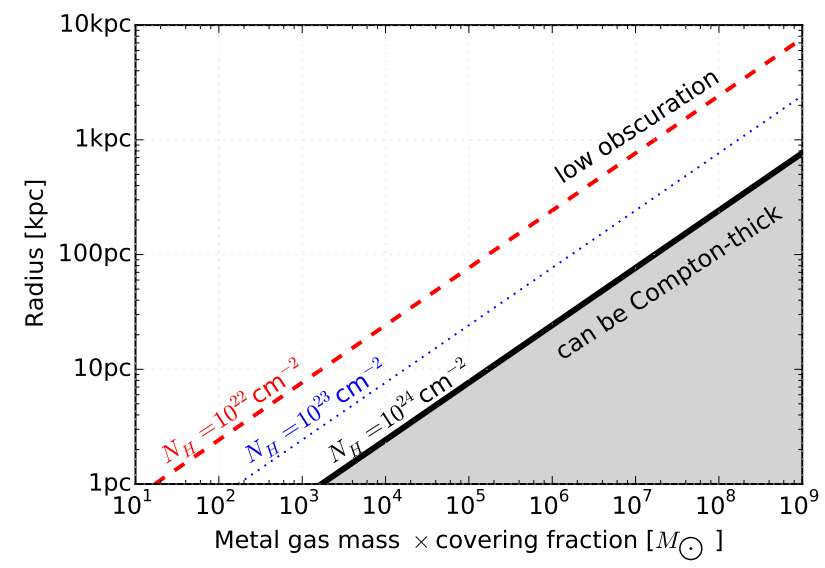

Figure B2. X-ray obscuration that a given metal gas mass can reach. An obscurer of column density $N_{\mathrm{H}}$ outside a radius $R$ has a minimum metal gas mass limit $M_{Z}$ (lines). For example, a $M_{Z}=$ $10^{9} M_{\odot}$ gas mass has to be brought withing $100 \mathrm{pc}$ to completely enshroud a region with Compton-thick column densities. Keep in mind that such masses are only found in very massive galaxies $\left(M_{\star}>3 \cdot 10^{10} M_{\odot}\right.$, see Figure B1). 